Supplement of Hydrol. Earth Syst. Sci., 24, 3111-3133, 2020

https://doi.org/10.5194/hess-24-3111-2020-supplement

(c) Author(s) 2020. This work is distributed under

the Creative Commons Attribution 4.0 License.

(c) (1)
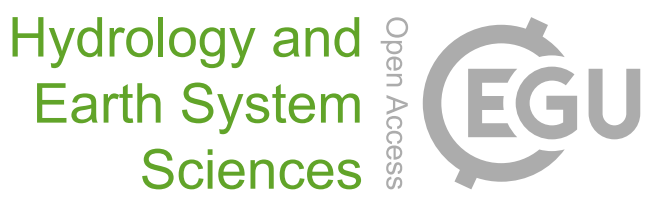

Supplement of

\title{
Crossing hydrological and geochemical modeling to understand the spatiotemporal variability of water chemistry in a headwater catchment (Strengbach, France)
}

Julien Ackerer et al.

Correspondence to: Julien Ackerer (julien.ackerer@ orange.fr), Benjamin Jeannot (bjeannot.pro@gmail.com), Frederick Delay (fdelay@unistra.fr), and François Chabaux (fchabaux@unistra.fr)

The copyright of individual parts of the supplement might differ from the CC BY 4.0 License. 


\section{Supplementary material, Figures}
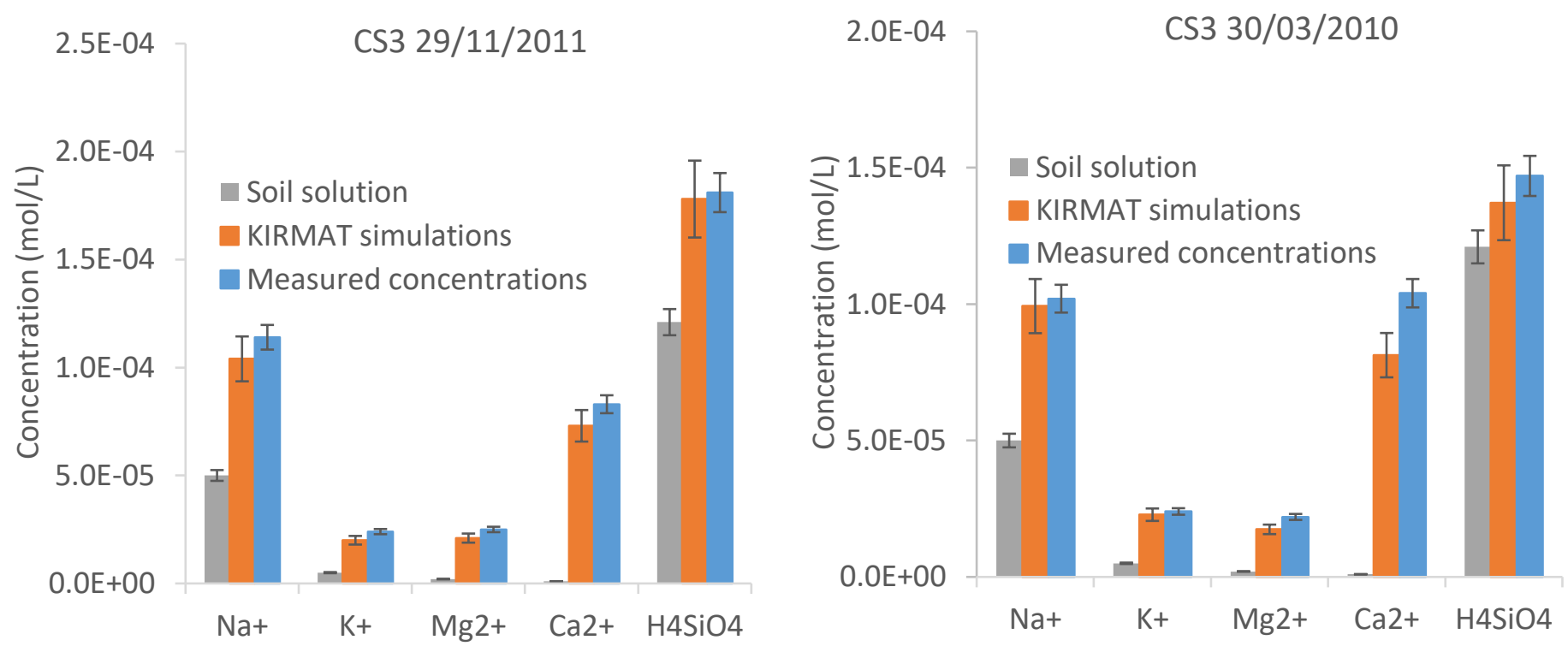

Figure S1: simulation results for the CS3 spring for an important drought (29/11/2011) and a strong flood event (30/03/2010). 

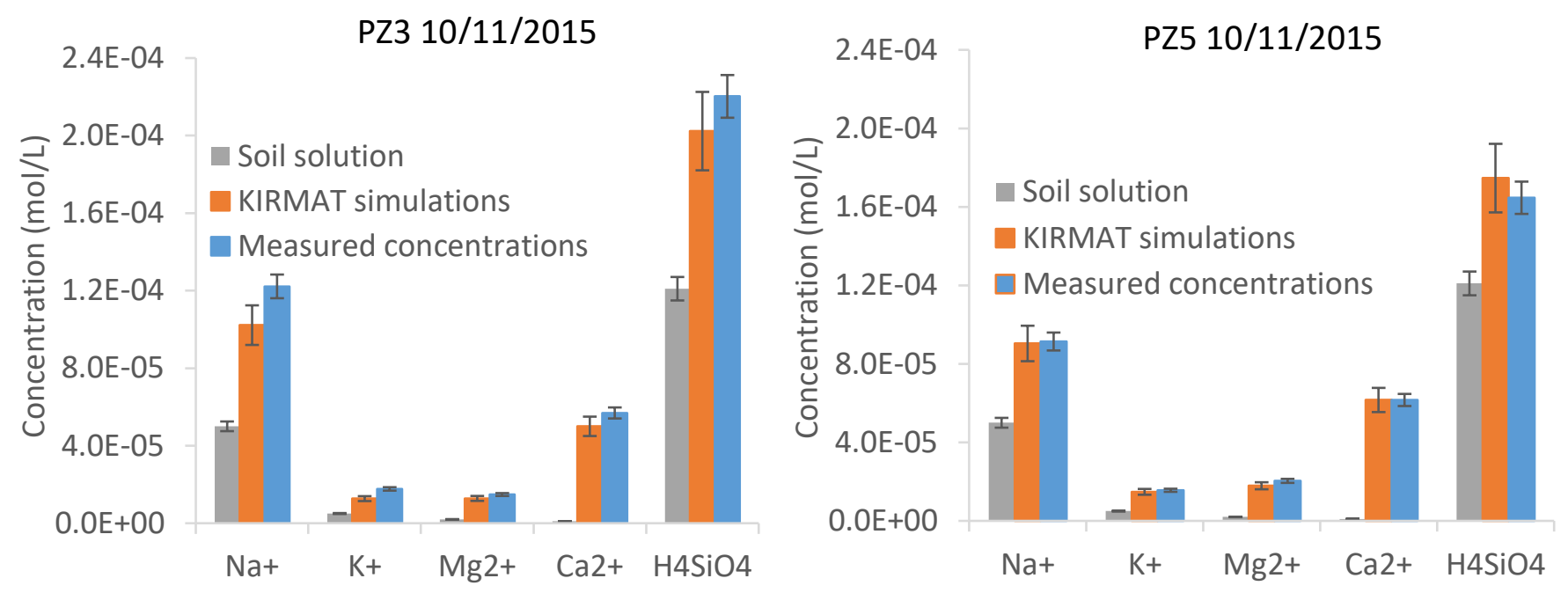

Figure S2: simulation results for the PZ3 and PZ5 piezometers for a drought period (10/11/2015). 
Supplementary material, Tables

\begin{tabular}{|c|c|c|c|c|c|c|c|c|c|c|}
\hline Date & $\mathrm{PH}$ & $\begin{array}{c}\text { Conductivity } \\
(\mu \mathrm{S} / \mathrm{cm})\end{array}$ & $\begin{array}{c}\mathrm{Na}^{+} \\
(\mathrm{mmol} / \mathrm{L})\end{array}$ & $\begin{array}{c}\mathrm{K}^{+} \\
(\mathrm{mmol} / \mathrm{L}) \\
\end{array}$ & $\begin{array}{c}\mathrm{Mg}^{2+} \\
(\mathrm{mmol} / \mathrm{L})\end{array}$ & $\begin{array}{c}\mathrm{Ca}^{2+} \\
(\mathrm{mmol} / \mathrm{L})\end{array}$ & $\begin{array}{c}\mathrm{Cl}^{-} \\
(\mathrm{mmol} / \mathrm{L})\end{array}$ & $\begin{array}{c}\mathrm{NO}_{3}^{-} \\
(\mathrm{mmol} / \mathrm{L}) \\
\end{array}$ & $\begin{array}{c}\mathrm{SO}_{4}{ }^{2-} \\
(\mathrm{mmol} / \mathrm{L})\end{array}$ & $\begin{array}{c}\mathrm{H}_{4} \mathrm{SiO}_{4} \\
(\mathrm{mmol} / \mathrm{L})\end{array}$ \\
\hline $14 / 01 / 1986$ & 5.99 & 47.00 & 0.087 & 0.020 & 0.031 & 0.102 & 0.047 & 0.070 & 0.117 & 0.141 \\
\hline $16 / 04 / 1986$ & 5.35 & 41.00 & 0.081 & 0.018 & 0.029 & 0.086 & 0.046 & 0.056 & 0.121 & 0.129 \\
\hline $14 / 05 / 1986$ & 5.35 & 43.00 & 0.080 & 0.018 & 0.028 & 0.083 & 0.045 & 0.061 & 0.112 & 0.127 \\
\hline 29/05/1986 & 5.60 & 39.00 & 0.084 & 0.018 & 0.029 & 0.088 & 0.045 & 0.060 & 0.111 & 0.131 \\
\hline 19/06/1986 & 5.55 & 39.00 & 0.084 & 0.017 & 0.029 & 0.088 & 0.047 & 0.063 & 0.112 & 0.134 \\
\hline 07/07/1986 & 5.85 & 42.30 & 0.090 & 0.018 & 0.029 & 0.095 & 0.047 & 0.062 & 0.111 & 0.145 \\
\hline $28 / 07 / 1986$ & 5.77 & 41.70 & 0.090 & 0.018 & 0.028 & 0.092 & 0.046 & 0.064 & 0.114 & 0.146 \\
\hline 01/09/1986 & 6.21 & 42.20 & 0.095 & 0.018 & 0.029 & 0.095 & 0.047 & 0.064 & 0.112 & 0.158 \\
\hline $15 / 09 / 1986$ & 6.56 & 44.60 & 0.094 & 0.018 & 0.030 & 0.103 & 0.048 & 0.069 & 0.110 & 0.160 \\
\hline $06 / 10 / 1986$ & 6.01 & 41.70 & 0.091 & 0.018 & 0.029 & 0.089 & 0.046 & 0.066 & 0.112 & 0.151 \\
\hline $27 / 10 / 1986$ & 5.44 & 42.50 & 0.085 & 0.019 & 0.029 & 0.089 & 0.050 & 0.069 & 0.116 & 0.137 \\
\hline 19/01/1987 & 5.51 & 39.20 & 0.088 & 0.017 & 0.030 & 0.087 & 0.045 & 0.064 & 0.110 & 0.146 \\
\hline 27/11/1989 & 6.10 & 38.00 & 0.088 & 0.017 & 0.025 & 0.085 & 0.049 & 0.052 & 0.099 & 0.156 \\
\hline $27 / 12 / 1989$ & 5.16 & 35.20 & 0.077 & 0.018 & 0.025 & 0.080 & 0.043 & 0.064 & 0.098 & 0.137 \\
\hline 08/01/1990 & 5.78 & 36.80 & 0.082 & 0.018 & 0.024 & 0.082 & 0.045 & 0.061 & 0.096 & 0.150 \\
\hline 19/02/1990 & 5.25 & 37.10 & 0.071 & 0.017 & 0.024 & 0.076 & 0.042 & 0.061 & 0.099 & 0.128 \\
\hline $13 / 08 / 1990$ & 6.22 & 38.00 & 0.092 & 0.019 & 0.024 & 0.082 & 0.051 & 0.057 & 0.095 & 0.164 \\
\hline $02 / 09 / 1991$ & 6.17 & 36.00 & 0.093 & 0.018 & 0.025 & 0.077 & 0.048 & 0.057 & 0.091 & 0.166 \\
\hline $23 / 09 / 1991$ & 6.63 & 38.60 & 0.090 & 0.020 & 0.026 & 0.099 & 0.050 & 0.070 & 0.089 & 0.168 \\
\hline $22 / 10 / 1991$ & 5.88 & 34.00 & 0.087 & 0.019 & 0.025 & 0.079 & 0.048 & 0.061 & 0.091 & 0.151 \\
\hline $11 / 10 / 1993$ & 5.80 & 33.00 & 0.080 & 0.016 & 0.022 & 0.076 & 0.046 & 0.058 & 0.089 & 0.145 \\
\hline $25 / 04 / 1994$ & 5.68 & 31.00 & 0.073 & 0.015 & 0.020 & 0.065 & 0.040 & 0.046 & 0.087 & 0.123 \\
\hline $18 / 05 / 1994$ & 5.76 & 34.40 & 0.078 & 0.016 & 0.021 & 0.065 & 0.040 & 0.046 & 0.064 & 0.140 \\
\hline $06 / 12 / 1994$ & 6.27 & 33.33 & 0.084 & 0.016 & 0.022 & 0.072 & 0.042 & 0.050 & 0.086 & 0.151 \\
\hline $24 / 03 / 1995$ & 5.26 & 32.70 & 0.073 & 0.016 & 0.020 & 0.065 & 0.041 & 0.049 & 0.085 & 0.129 \\
\hline $31 / 05 / 1995$ & 6.02 & 32.07 & 0.075 & 0.016 & 0.021 & 0.067 & 0.040 & 0.050 & 0.080 & 0.135 \\
\hline $18 / 07 / 1995$ & 6.19 & 32.52 & 0.082 & 0.015 & 0.021 & 0.066 & 0.038 & 0.048 & 0.083 & 0.151 \\
\hline $25 / 07 / 1995$ & 6.17 & 33.33 & 0.081 & 0.016 & 0.021 & 0.068 & 0.040 & 0.049 & 0.081 & 0.156 \\
\hline 23/07/1995 & 6.25 & 33.70 & 0.081 & 0.016 & 0.021 & 0.069 & 0.040 & 0.042 & 0.082 & 0.150 \\
\hline $13 / 12 / 1995$ & 6.12 & 33.61 & 0.082 & 0.017 & 0.021 & 0.067 & 0.041 & 0.050 & 0.083 & 0.150 \\
\hline $03 / 06 / 1997$ & 6.19 & 31.04 & 0.080 & 0.015 & 0.020 & 0.062 & 0.038 & 0.045 & 0.078 & 0.146 \\
\hline $12 / 07 / 2004$ & 5.90 & 28.20 & 0.078 & 0.018 & 0.015 & 0.051 & 0.043 & 0.027 & 0.062 & 0.158 \\
\hline $28 / 09 / 2004$ & 5.82 & 26.80 & 0.078 & 0.014 & 0.016 & 0.053 & 0.037 & 0.029 & 0.061 & 0.154 \\
\hline $13 / 12 / 2004$ & 6.12 & 24.50 & 0.073 & 0.013 & 0.014 & 0.048 & 0.039 & 0.027 & 0.060 & 0.145 \\
\hline $29 / 03 / 2005$ & 5.22 & 25.20 & 0.067 & 0.014 & 0.014 & 0.044 & 0.040 & 0.035 & 0.061 & 0.120 \\
\hline $03 / 05 / 2005$ & 6.19 & 22.10 & 0.068 & 0.012 & 0.013 & 0.043 & 0.036 & 0.024 & 0.058 & 0.133 \\
\hline $31 / 05 / 2005$ & 6.33 & 24.00 & 0.072 & 0.014 & 0.014 & 0.051 & 0.036 & 0.022 & 0.057 & 0.138 \\
\hline $11 / 07 / 2005$ & 6.44 & 25.50 & 0.076 & 0.014 & 0.014 & 0.053 & 0.036 & 0.024 & 0.059 & 0.157 \\
\hline $22 / 08 / 2005$ & 6.40 & 24.40 & 0.079 & 0.014 & 0.015 & 0.055 & 0.037 & 0.029 & 0.061 & 0.158 \\
\hline $03 / 10 / 2005$ & 6.50 & 24.00 & 0.075 & 0.014 & 0.015 & 0.049 & 0.046 & 0.026 & 0.058 & 0.151 \\
\hline $07 / 02 / 2006$ & 6.05 & 25.10 & 0.081 & 0.015 & 0.014 & 0.053 & 0.041 & 0.027 & 0.060 & 0.153 \\
\hline $03 / 04 / 2006$ & 5.33 & 25.50 & 0.068 & 0.015 & 0.015 & 0.044 & 0.044 & 0.038 & 0.062 & 0.113 \\
\hline $22 / 05 / 2006$ & 6.10 & 22.10 & 0.072 & 0.013 & 0.014 & 0.045 & 0.036 & 0.028 & 0.058 & 0.135 \\
\hline $10 / 07 / 2006$ & 6.48 & 25.80 & 0.075 & 0.013 & 0.014 & 0.047 & 0.035 & 0.022 & 0.057 & 0.153 \\
\hline $21 / 08 / 2006$ & 6.02 & 28.50 & 0.071 & 0.014 & 0.014 & 0.046 & 0.040 & 0.032 & 0.056 & 0.133 \\
\hline $02 / 10 / 2006$ & 5.85 & 25.70 & 0.071 & 0.013 & 0.014 & 0.044 & 0.044 & 0.033 & 0.057 & 0.135 \\
\hline $19 / 03 / 2007$ & 5.75 & 33.20 & 0.072 & 0.013 & 0.013 & 0.042 & 0.038 & 0.028 & 0.054 & 0.140 \\
\hline $16 / 04 / 2007$ & 5.78 & 24.50 & 0.068 & 0.012 & 0.013 & 0.042 & 0.038 & 0.027 & 0.053 & 0.136 \\
\hline $29 / 05 / 2007$ & 6.20 & 23.00 & 0.071 & 0.013 & 0.013 & 0.043 & 0.037 & 0.026 & 0.052 & 0.137 \\
\hline $23 / 07 / 2007$ & 6.63 & 22.90 & 0.072 & 0.013 & 0.014 & 0.044 & 0.038 & 0.028 & 0.053 & 0.142 \\
\hline $02 / 09 / 2007$ & 5.97 & 23.30 & 0.074 & 0.012 & 0.014 & 0.044 & 0.040 & 0.031 & 0.057 & 0.145 \\
\hline $15 / 10 / 2007$ & 6.46 & 23.90 & 0.075 & 0.013 & 0.040 & 0.045 & 0.040 & 0.029 & 0.053 & 0.149 \\
\hline $26 / 11 / 2007$ & 6.07 & 44.10 & 0.088 & 0.031 & 0.035 & 0.102 & 0.056 & 0.134 & 0.063 & 0.137 \\
\hline $21 / 01 / 2008$ & 5.85 & 23.30 & 0.072 & 0.013 & 0.014 & 0.042 & 0.041 & 0.030 & 0.052 & 0.140 \\
\hline $03 / 03 / 2008$ & 6.16 & 23.70 & 0.074 & 0.013 & 0.013 & 0.044 & 0.037 & 0.029 & 0.050 & 0.147 \\
\hline $14 / 04 / 2008$ & 5.77 & 24.40 & 0.070 & 0.014 & 0.015 & 0.042 & 0.043 & 0.040 & 0.054 & 0.117 \\
\hline
\end{tabular}




\begin{tabular}{|c|c|c|c|c|c|c|c|c|c|c|}
\hline $19 / 05 / 2008$ & 6.19 & 22.40 & 0.073 & 0.013 & 0.013 & 0.044 & 0.038 & 0.032 & 0.052 & 0.145 \\
\hline $22 / 05 / 2008$ & 6.08 & 21.60 & 0.074 & 0.013 & 0.013 & 0.043 & 0.038 & 0.029 & 0.050 & 0.145 \\
\hline $09 / 06 / 2008$ & 6.25 & 22.60 & 0.073 & 0.013 & 0.013 & 0.043 & 0.037 & 0.032 & 0.051 & 0.139 \\
\hline $04 / 08 / 2008$ & 6.66 & 23.40 & 0.079 & 0.013 & 0.015 & 0.044 & 0.035 & 0.029 & 0.050 & 0.158 \\
\hline $16 / 09 / 2008$ & 6.28 & 22.10 & 0.071 & 0.013 & 0.017 & 0.044 & 0.041 & 0.040 & 0.048 & 0.129 \\
\hline $27 / 10 / 2008$ & 6.23 & 24.10 & 0.075 & 0.014 & 0.014 & 0.043 & 0.040 & 0.035 & 0.047 & 0.138 \\
\hline $08 / 12 / 2008$ & 6.32 & 22.20 & 0.075 & 0.012 & 0.014 & 0.042 & 0.037 & 0.032 & 0.047 & 0.143 \\
\hline $19 / 01 / 2009$ & 6.17 & 23.30 & 0.075 & 0.012 & 0.014 & 0.037 & 0.037 & 0.031 & 0.047 & 0.147 \\
\hline $16 / 03 / 2009$ & 6.42 & 23.40 & 0.073 & 0.013 & 0.014 & 0.044 & 0.038 & 0.034 & 0.047 & 0.142 \\
\hline $11 / 05 / 2009$ & 6.26 & 23.00 & 0.075 & 0.012 & 0.013 & 0.046 & 0.040 & 0.029 & 0.055 & 0.140 \\
\hline $06 / 07 / 2009$ & 6.44 & 23.90 & 0.079 & 0.013 & 0.014 & 0.047 & 0.038 & 0.036 & 0.049 & 0.159 \\
\hline $01 / 09 / 2009$ & 6.45 & 25.40 & 0.080 & 0.012 & 0.013 & 0.043 & 0.038 & 0.034 & 0.049 & 0.160 \\
\hline $06 / 10 / 2009$ & 6.44 & 25.80 & 0.084 & 0.013 & 0.015 & 0.045 & 0.041 & 0.034 & 0.054 & 0.170 \\
\hline $08 / 12 / 2009$ & 6.46 & 23.60 & 0.078 & 0.013 & 0.013 & 0.044 & 0.039 & 0.041 & 0.045 & 0.151 \\
\hline $19 / 03 / 2010$ & 6.26 & 22.90 & 0.077 & 0.013 & 0.015 & 0.043 & 0.039 & 0.038 & 0.046 & 0.149 \\
\hline $30 / 03 / 2010$ & 5.61 & 24.40 & 0.074 & 0.014 & 0.015 & 0.043 & 0.049 & 0.055 & 0.047 & 0.120 \\
\hline $29 / 03 / 2011$ & 6.23 & 22.70 & 0.074 & 0.013 & 0.015 & 0.038 & 0.038 & 0.036 & 0.045 & 0.145 \\
\hline $24 / 05 / 2011$ & 6.78 & 23.00 & 0.079 & 0.012 & 0.016 & 0.036 & 0.037 & 0.032 & 0.048 & 0.158 \\
\hline $05 / 07 / 2011$ & 6.32 & 23.00 & 0.079 & 0.013 & 0.016 & 0.041 & 0.037 & 0.036 & 0.046 & 0.170 \\
\hline $02 / 08 / 2011$ & 6.33 & 32.00 & 0.075 & 0.013 & 0.016 & 0.035 & 0.040 & 0.039 & 0.044 & 0.168 \\
\hline $30 / 08 / 2011$ & 6.46 & 22.50 & 0.076 & 0.012 & 0.014 & 0.040 & 0.037 & 0.036 & 0.044 & 0.164 \\
\hline $04 / 10 / 2011$ & 6.57 & 23.90 & 0.080 & 0.012 & 0.016 & 0.042 & 0.040 & 0.036 & 0.045 & 0.176 \\
\hline $25 / 10 / 2011$ & 6.34 & 23.60 & 0.084 & 0.017 & 0.015 & 0.033 & 0.043 & 0.039 & 0.045 & 0.163 \\
\hline $29 / 11 / 2011$ & 6.30 & 23.00 & 0.088 & 0.015 & 0.019 & 0.034 & 0.037 & 0.033 & 0.047 & 0.177 \\
\hline $05 / 05 / 2015$ & 5.33 & 23.40 & 0.065 & 0.012 & 0.012 & 0.054 & 0.039 & 0.060 & 0.040 & 0.121 \\
\hline $26 / 08 / 2015$ & 6.33 & 32.00 & 0.082 & 0.012 & 0.013 & 0.042 & 0.039 & 0.046 & 0.041 & 0.172 \\
\hline $10 / 11 / 2015$ & 6.42 & 24.42 & 0.084 & 0.011 & 0.014 & 0.046 & 0.041 & 0.045 & 0.046 & 0.178 \\
\hline $02 / 12 / 2015$ & 5.73 & 24.54 & 0.071 & 0.014 & 0.015 & 0.046 & 0.043 & 0.068 & 0.039 & 0.129 \\
\hline
\end{tabular}

Table S1: chemical data for the CS1 spring from 1986 to 2015. 


\begin{tabular}{|c|c|c|c|c|c|c|c|c|c|c|}
\hline Date & PH & $\begin{array}{c}\text { Conductivity } \\
(\mu \mathrm{S} / \mathrm{cm})\end{array}$ & $\begin{array}{c}\mathrm{Na}^{+} \\
(\mathrm{mmol} / \mathrm{L})\end{array}$ & $\begin{array}{c}\mathrm{K}^{+} \\
(\mathrm{mmol} / \mathrm{L})\end{array}$ & $\begin{array}{c}\mathrm{Mg}^{2+} \\
(\mathrm{mmol} / \mathrm{L})\end{array}$ & $\begin{array}{c}\mathrm{Ca}^{2+} \\
(\mathrm{mmol} / \mathrm{L})\end{array}$ & $\begin{array}{c}\mathrm{Cl}^{-} \\
(\mathrm{mmol} / \mathrm{L})\end{array}$ & $\begin{array}{c}\mathrm{NO}_{3}^{-} \\
(\mathrm{mmol} / \mathrm{L})\end{array}$ & $\begin{array}{c}\mathrm{SO}_{4}^{2-} \\
(\mathrm{mmol} / \mathrm{L})\end{array}$ & $\begin{array}{c}\mathrm{H}_{4} \mathrm{SiO}_{4} \\
(\mathrm{mmol} / \mathrm{L})\end{array}$ \\
\hline $14 / 01 / 1986$ & 6.16 & 44.00 & 0.096 & 0.021 & 0.026 & 0.095 & 0.052 & 0.040 & 0.100 & 0.141 \\
\hline $16 / 04 / 1986$ & 5.98 & 39.00 & 0.080 & 0.021 & 0.024 & 0.091 & 0.047 & 0.000 & 0.102 & 0.118 \\
\hline $14 / 05 / 1986$ & 5.78 & 40.00 & 0.076 & 0.019 & 0.024 & 0.089 & 0.046 & 0.032 & 0.102 & 0.112 \\
\hline 29/05/1986 & 6.28 & 37.00 & 0.082 & 0.018 & 0.024 & 0.088 & 0.049 & 0.038 & 0.100 & 0.120 \\
\hline $19 / 06 / 1986$ & 6.19 & 38.00 & 0.082 & 0.019 & 0.026 & 0.091 & 0.048 & 0.038 & 0.102 & 0.122 \\
\hline $07 / 07 / 1986$ & 6.02 & 38.50 & 0.086 & 0.019 & 0.024 & 0.088 & 0.052 & 0.029 & 0.099 & 0.134 \\
\hline $28 / 07 / 1986$ & 5.85 & 39.20 & 0.087 & 0.019 & 0.024 & 0.090 & 0.050 & 0.038 & 0.100 & 0.138 \\
\hline $01 / 09 / 1986$ & 6.49 & 39.10 & 0.093 & 0.020 & 0.024 & 0.089 & 0.048 & 0.048 & 0.098 & 0.152 \\
\hline $15 / 09 / 1986$ & 6.51 & 39.70 & 0.092 & 0.020 & 0.024 & 0.094 & 0.047 & 0.036 & 0.095 & 0.154 \\
\hline $06 / 10 / 1986$ & 6.41 & 40.30 & 0.088 & 0.020 & 0.024 & 0.093 & 0.048 & 0.039 & 0.101 & 0.143 \\
\hline $27 / 10 / 1986$ & 6.26 & 40.30 & 0.087 & 0.021 & 0.024 & 0.091 & 0.048 & 0.054 & 0.102 & 0.134 \\
\hline $19 / 01 / 1987$ & 6.27 & 36.60 & 0.085 & 0.019 & 0.024 & 0.088 & 0.048 & 0.049 & 0.099 & 0.134 \\
\hline $27 / 11 / 1989$ & 6.35 & 37.00 & 0.087 & 0.019 & 0.022 & 0.087 & 0.047 & 0.039 & 0.093 & 0.149 \\
\hline $27 / 12 / 1989$ & 5.56 & 35.50 & 0.080 & 0.020 & 0.022 & 0.091 & 0.042 & 0.051 & 0.090 & 0.128 \\
\hline 08/01/1990 & 5.94 & 36.10 & 0.079 & 0.020 & 0.020 & 0.088 & 0.046 & 0.052 & 0.090 & 0.138 \\
\hline $19 / 02 / 1990$ & 6.13 & 38.50 & 0.073 & 0.020 & 0.022 & 0.093 & 0.047 & 0.062 & 0.098 & 0.114 \\
\hline $13 / 08 / 1990$ & 6.25 & 36.00 & 0.091 & 0.019 & 0.021 & 0.084 & 0.048 & 0.044 & 0.091 & 0.159 \\
\hline $02 / 09 / 1991$ & 6.41 & 34.00 & 0.093 & 0.020 & 0.020 & 0.078 & 0.046 & 0.049 & 0.088 & 0.165 \\
\hline $23 / 09 / 1991$ & 6.22 & 34.60 & 0.093 & 0.020 & 0.020 & 0.078 & 0.047 & 0.045 & 0.083 & 0.163 \\
\hline $11 / 10 / 1993$ & 6.43 & 35.00 & 0.086 & 0.019 & 0.022 & 0.092 & 0.048 & 0.046 & 0.089 & 0.143 \\
\hline $25 / 04 / 1994$ & 5.76 & 31.00 & 0.075 & 0.018 & 0.020 & 0.079 & 0.041 & 0.037 & 0.090 & 0.117 \\
\hline $18 / 05 / 1994$ & 6.00 & 36.10 & 0.080 & 0.018 & 0.020 & 0.075 & 0.043 & 0.038 & 0.090 & 0.134 \\
\hline $06 / 12 / 1994$ & 6.44 & 36.14 & 0.092 & 0.019 & 0.021 & 0.083 & 0.048 & 0.045 & 0.087 & 0.149 \\
\hline $24 / 03 / 1995$ & 5.53 & 34.42 & 0.078 & 0.019 & 0.021 & 0.081 & 0.046 & 0.046 & 0.087 & 0.121 \\
\hline $31 / 05 / 1995$ & 6.43 & 34.51 & 0.081 & 0.018 & 0.020 & 0.077 & 0.044 & 0.041 & 0.085 & 0.128 \\
\hline $18 / 07 / 1995$ & 6.34 & 34.87 & 0.086 & 0.018 & 0.020 & 0.075 & 0.045 & 0.044 & 0.085 & 0.147 \\
\hline $23 / 07 / 1995$ & 6.30 & 34.69 & 0.084 & 0.018 & 0.020 & 0.072 & 0.048 & 0.021 & 0.081 & 0.143 \\
\hline $25 / 07 / 1995$ & 6.41 & 35.06 & 0.085 & 0.018 & 0.020 & 0.075 & 0.045 & 0.042 & 0.084 & 0.151 \\
\hline $13 / 12 / 1995$ & 6.35 & 37.05 & 0.089 & 0.020 & 0.021 & 0.079 & 0.050 & 0.048 & 0.084 & 0.147 \\
\hline $12 / 07 / 2004$ & 5.56 & 34.60 & 0.091 & 0.018 & 0.018 & 0.068 & 0.051 & 0.054 & 0.066 & 0.157 \\
\hline $28 / 09 / 2004$ & 5.58 & 34.40 & 0.094 & 0.018 & 0.019 & 0.070 & 0.052 & 0.058 & 0.064 & 0.155 \\
\hline $13 / 12 / 2004$ & 6.44 & 31.90 & 0.086 & 0.017 & 0.018 & 0.071 & 0.055 & 0.063 & 0.063 & 0.138 \\
\hline $03 / 05 / 2005$ & 6.23 & 28.80 & 0.078 & 0.016 & 0.017 & 0.067 & 0.050 & 0.050 & 0.060 & 0.124 \\
\hline $31 / 05 / 2005$ & 6.30 & 30.20 & 0.082 & 0.017 & 0.017 & 0.070 & 0.050 & 0.055 & 0.061 & 0.131 \\
\hline $11 / 07 / 2005$ & 6.27 & 30.50 & 0.087 & 0.016 & 0.017 & 0.067 & 0.049 & 0.054 & 0.060 & 0.149 \\
\hline $22 / 08 / 2005$ & 6.47 & 29.50 & 0.093 & 0.017 & 0.017 & 0.069 & 0.049 & 0.061 & 0.063 & 0.157 \\
\hline $07 / 02 / 2006$ & 6.24 & 32.30 & 0.092 & 0.019 & 0.018 & 0.069 & 0.055 & 0.060 & 0.062 & 0.151 \\
\hline $03 / 04 / 2006$ & 6.04 & 31.20 & 0.073 & 0.018 & 0.018 & 0.073 & 0.049 & 0.067 & 0.064 & 0.107 \\
\hline $22 / 05 / 2006$ & 6.28 & 28.70 & 0.084 & 0.017 & 0.018 & 0.067 & 0.051 & 0.061 & 0.060 & 0.128 \\
\hline $10 / 07 / 2006$ & 6.50 & 32.60 & 0.087 & 0.018 & 0.017 & 0.067 & 0.047 & 0.056 & 0.059 & 0.146 \\
\hline $21 / 08 / 2006$ & 6.30 & 33.10 & 0.084 & 0.017 & 0.018 & 0.068 & 0.056 & 0.063 & 0.058 & 0.134 \\
\hline $02 / 10 / 2006$ & 6.30 & 32.30 & 0.082 & 0.023 & 0.018 & 0.072 & 0.048 & 0.065 & 0.057 & 0.129 \\
\hline $19 / 03 / 2007$ & 5.61 & 32.00 & 0.079 & 0.017 & 0.017 & 0.063 & 0.048 & 0.058 & 0.058 & 0.132 \\
\hline $16 / 04 / 2007$ & 5.57 & 32.00 & 0.077 & 0.017 & 0.016 & 0.064 & 0.047 & 0.067 & 0.058 & 0.130 \\
\hline $19 / 05 / 2008$ & 6.12 & 29.10 & 0.083 & 0.017 & 0.017 & 0.065 & 0.048 & 0.066 & 0.056 & 0.139 \\
\hline $11 / 05 / 2009$ & 6.27 & 31.10 & 0.086 & 0.015 & 0.018 & 0.070 & 0.050 & 0.080 & 0.050 & 0.134 \\
\hline $06 / 07 / 2009$ & 6.27 & 30.20 & 0.090 & 0.017 & 0.017 & 0.067 & 0.047 & 0.074 & 0.050 & 0.156 \\
\hline $06 / 10 / 2009$ & 6.37 & 33.90 & 0.097 & 0.018 & 0.018 & 0.064 & 0.051 & 0.075 & 0.056 & 0.171 \\
\hline $08 / 12 / 2009$ & 6.62 & 31.50 & 0.094 & 0.017 & 0.019 & 0.068 & 0.049 & 0.079 & 0.050 & 0.154 \\
\hline $19 / 03 / 2010$ & 6.26 & 31.70 & 0.090 & 0.017 & 0.019 & 0.066 & 0.051 & 0.094 & 0.047 & 0.142 \\
\hline $30 / 03 / 2010$ & 6.15 & 34.70 & 0.086 & 0.019 & 0.022 & 0.080 & 0.056 & 0.129 & 0.045 & 0.122 \\
\hline $29 / 03 / 2011$ & 6.18 & 32.40 & 0.091 & 0.018 & 0.021 & 0.065 & 0.050 & 0.099 & 0.046 & 0.144 \\
\hline $24 / 05 / 2011$ & 6.52 & 31.00 & 0.098 & 0.022 & 0.019 & 0.058 & 0.052 & 0.086 & 0.046 & 0.158 \\
\hline $05 / 07 / 2011$ & 6.29 & 31.50 & 0.100 & 0.017 & 0.021 & 0.065 & 0.049 & 0.095 & 0.047 & 0.171 \\
\hline $02 / 08 / 2011$ & 6.50 & 30.80 & 0.089 & 0.018 & 0.021 & 0.063 & 0.048 & 0.102 & 0.042 & 0.170 \\
\hline $30 / 08 / 2011$ & 6.55 & 31.60 & 0.090 & 0.017 & 0.020 & 0.066 & 0.050 & 0.103 & 0.044 & 0.159 \\
\hline
\end{tabular}




\begin{tabular}{|l|l|l|l|l|l|l|l|l|l|l|}
\hline $04 / 10 / 2011$ & 6.76 & 33.37 & 0.098 & 0.017 & 0.018 & 0.065 & 0.051 & 0.095 & 0.045 & 0.177 \\
\hline $25 / 10 / 2011$ & 6.42 & 32.35 & 0.099 & 0.021 & 0.019 & 0.056 & 0.051 & 0.094 & 0.044 & 0.171 \\
\hline $29 / 11 / 2011$ & 6.22 & 32.70 & 0.102 & 0.020 & 0.024 & 0.058 & 0.050 & 0.094 & 0.048 & 0.180 \\
\hline $05 / 05 / 2015$ & 6.14 & 30.70 & 0.077 & 0.016 & 0.018 & 0.074 & 0.039 & 0.095 & 0.044 & 0.123 \\
\hline $26 / 08 / 2015$ & 6.34 & 30.50 & 0.093 & 0.017 & 0.017 & 0.061 & 0.043 & 0.079 & 0.044 & 0.170 \\
\hline $10 / 11 / 2015$ & 6.58 & 30.38 & 0.096 & 0.015 & 0.017 & 0.062 & 0.042 & 0.077 & 0.045 & 0.180 \\
\hline
\end{tabular}

Table S2: chemical data for the CS2 spring from 1986 to 2015. 


\begin{tabular}{|c|c|c|c|c|c|c|c|c|c|c|}
\hline Date & $\mathrm{PH}$ & $\begin{array}{c}\text { Conductivity } \\
(\mu \mathrm{S} / \mathrm{cm})\end{array}$ & $\begin{array}{c}\mathrm{Na}^{+} \\
(\mathrm{mmol} / \mathrm{L})\end{array}$ & $\begin{array}{c}\mathrm{K}^{+} \\
(\mathrm{mmol} / \mathrm{L})\end{array}$ & $\begin{array}{c}\mathrm{Mg}^{2+} \\
(\mathrm{mmol} / \mathrm{L})\end{array}$ & $\begin{array}{c}\mathrm{Ca}^{2+} \\
(\mathrm{mmol} / \mathrm{L})\end{array}$ & $\begin{array}{c}\mathrm{Cl}^{-} \\
(\mathrm{mmol} / \mathrm{L})\end{array}$ & $\begin{array}{c}\mathrm{NO}_{3}^{-} \\
(\mathrm{mmol} / \mathrm{L})\end{array}$ & $\begin{array}{c}\mathrm{SO}_{4}^{2-} \\
(\mathrm{mmol} / \mathrm{L})\end{array}$ & $\begin{array}{c}\mathrm{H}_{4} \mathrm{SiO}_{4} \\
(\mathrm{mmol} / \mathrm{L})\end{array}$ \\
\hline $14 / 01 / 1986$ & 6.43 & 47.00 & 0.104 & 0.025 & 0.022 & 0.113 & 0.047 & 0.050 & 0.104 & 0.154 \\
\hline $16 / 04 / 1986$ & 6.25 & 42.00 & 0.099 & 0.024 & 0.022 & 0.103 & 0.052 & 0.032 & 0.108 & 0.147 \\
\hline $14 / 05 / 1986$ & 6.21 & 41.00 & 0.093 & 0.023 & 0.020 & 0.097 & 0.049 & 0.016 & 0.106 & 0.141 \\
\hline $29 / 05 / 1986$ & 6.48 & 38.00 & 0.097 & 0.023 & 0.021 & 0.096 & 0.045 & 0.043 & 0.102 & 0.148 \\
\hline $19 / 06 / 1986$ & 6.32 & 40.00 & 0.099 & 0.023 & 0.021 & 0.094 & 0.048 & 0.036 & 0.102 & 0.150 \\
\hline $07 / 07 / 1986$ & 6.23 & 40.60 & 0.101 & 0.024 & 0.020 & 0.097 & 0.047 & 0.045 & 0.101 & 0.150 \\
\hline $28 / 07 / 1986$ & 6.12 & 39.00 & 0.099 & 0.023 & 0.019 & 0.091 & 0.045 & 0.041 & 0.099 & 0.156 \\
\hline $01 / 09 / 1986$ & 6.48 & 37.70 & 0.099 & 0.022 & 0.019 & 0.088 & 0.045 & 0.030 & 0.091 & 0.162 \\
\hline $15 / 09 / 1986$ & 6.58 & 38.00 & 0.099 & 0.022 & 0.018 & 0.090 & 0.045 & 0.014 & 0.089 & 0.161 \\
\hline $06 / 10 / 1986$ & 6.56 & 39.20 & 0.100 & 0.023 & 0.019 & 0.086 & 0.045 & 0.045 & 0.098 & 0.160 \\
\hline $27 / 10 / 1986$ & 6.54 & 42.50 & 0.101 & 0.025 & 0.020 & 0.095 & 0.048 & 0.070 & 0.103 & 0.157 \\
\hline $19 / 01 / 1987$ & 6.27 & 39.60 & 0.098 & 0.023 & 0.021 & 0.096 & 0.049 & 0.061 & 0.102 & 0.156 \\
\hline $27 / 11 / 1989$ & 6.32 & 37.00 & 0.100 & 0.023 & 0.017 & 0.090 & 0.045 & 0.030 & 0.094 & 0.164 \\
\hline $27 / 12 / 1989$ & 6.05 & 40.69 & 0.096 & 0.025 & 0.020 & 0.105 & 0.049 & 0.075 & 0.103 & 0.155 \\
\hline $08 / 01 / 1990$ & 6.28 & 39.30 & 0.096 & 0.024 & 0.018 & 0.098 & 0.047 & 0.063 & 0.098 & 0.161 \\
\hline $08 / 01 / 1990$ & 6.13 & 44.10 & 0.092 & 0.024 & 0.022 & 0.113 & 0.052 & 0.088 & 0.107 & 0.138 \\
\hline $13 / 08 / 1990$ & 6.39 & 37.00 & 0.099 & 0.022 & 0.017 & 0.087 & 0.046 & 0.045 & 0.091 & 0.169 \\
\hline $03 / 12 / 1990$ & 6.20 & - & 0.098 & 0.026 & 0.019 & 0.093 & 0.047 & 0.062 & 0.094 & 0.163 \\
\hline $17 / 12 / 1990$ & 6.47 & 39.00 & 0.094 & 0.023 & 0.017 & 0.088 & 0.047 & 0.058 & 0.091 & 0.165 \\
\hline $31 / 12 / 1990$ & 6.31 & 46.00 & 0.094 & 0.025 & 0.023 & 0.122 & 0.049 & 0.083 & 0.098 & 0.149 \\
\hline $14 / 01 / 1991$ & 6.11 & 49.00 & 0.095 & 0.025 & 0.022 & 0.110 & 0.053 & 0.077 & 0.103 & 0.144 \\
\hline $29 / 01 / 1991$ & 6.21 & 39.00 & 0.095 & 0.024 & 0.018 & 0.091 & 0.046 & 0.059 & 0.094 & 0.157 \\
\hline $11 / 02 / 1991$ & 6.26 & 38.10 & 0.093 & 0.024 & 0.017 & 0.088 & 0.043 & 0.054 & 0.092 & 0.161 \\
\hline $25 / 02 / 1991$ & 6.34 & 36.60 & 0.094 & 0.023 & 0.017 & 0.086 & 0.044 & 0.050 & 0.090 & 0.162 \\
\hline $11 / 03 / 1991$ & 6.32 & 39.00 & 0.096 & 0.023 & 0.018 & 0.090 & 0.045 & 0.056 & 0.092 & 0.163 \\
\hline $25 / 03 / 1991$ & 6.15 & 39.00 & 0.097 & 0.023 & 0.018 & 0.090 & 0.046 & 0.054 & 0.092 & 0.161 \\
\hline $08 / 04 / 1991$ & 6.45 & 38.00 & 0.096 & 0.023 & 0.019 & 0.095 & 0.049 & 0.053 & 0.094 & 0.161 \\
\hline $06 / 05 / 1991$ & 6.36 & 36.30 & 0.098 & 0.023 & 0.018 & 0.086 & 0.044 & 0.051 & 0.089 & 0.163 \\
\hline $03 / 06 / 1991$ & 6.18 & 33.00 & 0.097 & 0.024 & 0.018 & 0.088 & 0.054 & 0.049 & 0.090 & 0.162 \\
\hline $02 / 09 / 1991$ & 6.34 & - & 0.096 & 0.024 & 0.017 & 0.079 & 0.043 & 0.042 & 0.087 & 0.168 \\
\hline $22 / 04 / 1991$ & 6.27 & 37.00 & 0.095 & 0.023 & 0.018 & 0.089 & 0.045 & 0.055 & 0.092 & 0.163 \\
\hline $17 / 06 / 1991$ & 6.56 & 38.00 & 0.098 & 0.024 & 0.017 & 0.084 & 0.044 & 0.052 & 0.090 & 0.162 \\
\hline $01 / 07 / 1991$ & 6.31 & 38.00 & 0.097 & 0.024 & 0.017 & 0.085 & 0.044 & 0.042 & 0.089 & 0.164 \\
\hline $15 / 07 / 1991$ & 5.87 & 34.70 & 0.092 & 0.023 & 0.023 & 0.085 & 0.047 & 0.045 & 0.090 & 0.156 \\
\hline $29 / 07 / 1991$ & 6.32 & 36.00 & 0.098 & 0.025 & 0.017 & 0.086 & 0.044 & 0.041 & 0.088 & 0.164 \\
\hline $12 / 08 / 1991$ & 6.41 & 36.00 & 0.098 & 0.025 & 0.017 & 0.082 & 0.043 & 0.047 & 0.089 & 0.165 \\
\hline $26 / 08 / 1991$ & 6.38 & 35.00 & 0.097 & 0.024 & 0.017 & 0.082 & 0.044 & 0.046 & 0.089 & 0.168 \\
\hline $02 / 09 / 1991$ & 6.34 & 35.00 & 0.096 & 0.024 & 0.017 & 0.079 & 0.043 & 0.042 & 0.087 & 0.168 \\
\hline $09 / 09 / 1991$ & 6.39 & 35.00 & 0.097 & 0.023 & 0.017 & 0.079 & 0.042 & 0.044 & 0.087 & 0.167 \\
\hline $23 / 09 / 1991$ & 6.43 & 36.30 & 0.098 & 0.024 & 0.017 & 0.086 & 0.044 & 0.056 & 0.087 & 0.167 \\
\hline $07 / 10 / 1991$ & 6.35 & 36.00 & 0.100 & 0.025 & 0.018 & 0.087 & 0.044 & 0.056 & 0.087 & 0.165 \\
\hline $21 / 10 / 1991$ & 6.22 & 36.00 & 0.101 & 0.025 & 0.018 & 0.089 & 0.045 & 0.058 & 0.087 & 0.165 \\
\hline $04 / 11 / 1991$ & 6.45 & 37.00 & 0.099 & 0.025 & 0.018 & 0.096 & 0.046 & 0.061 & 0.090 & 0.164 \\
\hline $18 / 11 / 1991$ & 6.43 & 40.00 & 0.101 & 0.025 & 0.020 & 0.100 & 0.051 & 0.069 & 0.099 & 0.156 \\
\hline $02 / 12 / 1991$ & 6.48 & 43.00 & 0.103 & 0.024 & 0.020 & 0.094 & 0.050 & 0.063 & 0.096 & 0.163 \\
\hline $16 / 12 / 1991$ & 6.42 & 38.00 & 0.101 & 0.023 & 0.018 & 0.088 & 0.047 & 0.059 & 0.093 & 0.165 \\
\hline $23 / 12 / 1991$ & 6.41 & 44.00 & 0.085 & 0.024 & 0.023 & 0.133 & 0.061 & 0.073 & 0.116 & 0.109 \\
\hline $03 / 02 / 1992$ & 6.50 & 36.00 & 0.098 & 0.023 & 0.018 & 0.091 & 0.047 & 0.047 & 0.093 & 0.164 \\
\hline $06 / 01 / 1992$ & 6.28 & 40.00 & 0.099 & 0.024 & 0.020 & 0.097 & 0.050 & 0.059 & 0.102 & 0.158 \\
\hline $16 / 03 / 1992$ & 6.24 & 41.00 & 0.092 & 0.025 & 0.021 & 0.114 & 0.050 & 0.064 & 0.105 & 0.140 \\
\hline $23 / 03 / 1992$ & 6.38 & 40.00 & 0.096 & 0.025 & 0.021 & 0.104 & 0.057 & 0.067 & 0.104 & 0.149 \\
\hline $30 / 03 / 1992$ & 6.46 & 39.00 & 0.095 & 0.024 & 0.020 & 0.104 & 0.049 & 0.063 & 0.103 & 0.153 \\
\hline $27 / 04 / 1992$ & 6.31 & 37.43 & 0.097 & 0.025 & 0.020 & 0.095 & 0.049 & 0.052 & 0.103 & 0.149 \\
\hline $25 / 05 / 1992$ & 6.29 & 36.05 & 0.096 & 0.015 & 0.018 & 0.083 & 0.045 & 0.017 & 0.095 & 0.155 \\
\hline $09 / 06 / 1992$ & 5.81 & 34.97 & 0.094 & 0.016 & 0.018 & 0.083 & 0.046 & 0.022 & 0.094 & 0.157 \\
\hline $22 / 06 / 1992$ & 6.20 & 34.28 & 0.094 & 0.020 & 0.016 & 0.082 & 0.045 & 0.021 & 0.094 & 0.158 \\
\hline $06 / 07 / 1992$ & 6.50 & 36.00 & 0.097 & 0.023 & 0.017 & 0.085 & 0.044 & 0.045 & 0.091 & 0.164 \\
\hline $20 / 07 / 1992$ & 6.48 & 34.00 & 0.098 & 0.024 & 0.017 & 0.085 & 0.045 & 0.043 & 0.091 & 0.166 \\
\hline
\end{tabular}




\begin{tabular}{|c|c|c|c|c|c|c|c|c|c|c|}
\hline 03/08/1992 & 6.68 & 36.00 & 0.098 & 0.024 & 0.017 & 0.086 & 0.044 & 0.039 & 0.089 & 0.163 \\
\hline $17 / 08 / 1992$ & 6.06 & 36.00 & 0.095 & 0.024 & 0.017 & 0.085 & 0.043 & 0.044 & 0.089 & 0.164 \\
\hline 01/09/1992 & 6.11 & 36.00 & 0.094 & 0.023 & 0.017 & 0.084 & 0.044 & 0.042 & 0.089 & 0.164 \\
\hline 07/09/1992 & 6.47 & 35.00 & 0.095 & 0.023 & 0.017 & 0.086 & 0.045 & 0.045 & 0.088 & 0.165 \\
\hline $14 / 09 / 1992$ & 6.36 & 35.00 & 0.097 & 0.023 & 0.017 & 0.085 & 0.044 & 0.047 & 0.088 & 0.166 \\
\hline $12 / 10 / 1992$ & 6.40 & 34.00 & 0.098 & 0.022 & 0.016 & 0.085 & 0.043 & 0.046 & 0.088 & 0.166 \\
\hline $02 / 11 / 1992$ & 6.50 & 39.00 & 0.101 & 0.023 & 0.020 & 0.102 & 0.049 & 0.064 & 0.100 & 0.157 \\
\hline 09/11/1992 & 6.50 & 39.00 & 0.101 & 0.023 & 0.018 & 0.095 & 0.047 & 0.058 & 0.095 & 0.159 \\
\hline $23 / 11 / 1992$ & 6.30 & 42.05 & 0.091 & 0.025 & 0.022 & 0.114 & 0.052 & 0.060 & 0.111 & 0.129 \\
\hline 07/12/1992 & 6.30 & 37.05 & 0.095 & 0.025 & 0.020 & 0.099 & 0.050 & 0.057 & 0.100 & 0.153 \\
\hline $04 / 01 / 1993$ & 6.42 & 38.05 & 0.098 & 0.025 & 0.018 & 0.091 & 0.047 & 0.052 & 0.095 & 0.157 \\
\hline $18 / 01 / 1993$ & 6.49 & 37.95 & 0.096 & 0.022 & 0.019 & 0.089 & 0.047 & 0.053 & 0.095 & 0.160 \\
\hline 01/02/1993 & 6.35 & 39.05 & 0.099 & 0.025 & 0.019 & 0.097 & 0.049 & 0.048 & 0.100 & 0.153 \\
\hline $15 / 02 / 1993$ & 6.61 & 38.05 & 0.097 & 0.024 & 0.018 & 0.090 & 0.046 & 0.052 & 0.096 & 0.157 \\
\hline $01 / 03 / 1993$ & 6.41 & 35.05 & 0.098 & 0.024 & 0.018 & 0.089 & 0.046 & 0.038 & 0.093 & 0.158 \\
\hline $15 / 03 / 1993$ & 6.36 & 36.05 & 0.096 & 0.023 & 0.017 & 0.087 & 0.045 & 0.045 & 0.091 & 0.162 \\
\hline 29/03/1993 & 6.39 & 35.05 & 0.096 & 0.024 & 0.018 & 0.087 & 0.045 & 0.047 & 0.092 & 0.163 \\
\hline $13 / 04 / 1993$ & 6.36 & 36.05 & 0.096 & 0.024 & 0.017 & 0.086 & 0.046 & 0.038 & 0.093 & 0.161 \\
\hline $26 / 04 / 1993$ & 6.37 & 35.05 & 0.099 & 0.022 & 0.017 & 0.084 & 0.045 & 0.048 & 0.092 & 0.161 \\
\hline $10 / 05 / 1993$ & 6.40 & 37.05 & 0.099 & 0.022 & 0.017 & 0.088 & 0.048 & 0.045 & 0.094 & 0.160 \\
\hline $24 / 05 / 1993$ & 6.31 & 36.05 & 0.098 & 0.022 & 0.017 & 0.086 & 0.044 & 0.047 & 0.093 & 0.161 \\
\hline 07/06/1993 & 6.43 & 34.04 & 0.098 & 0.023 & 0.017 & 0.087 & 0.044 & 0.021 & 0.089 & 0.168 \\
\hline $21 / 06 / 1993$ & 6.38 & 37.05 & 0.097 & 0.023 & 0.017 & 0.085 & 0.044 & 0.024 & 0.089 & 0.168 \\
\hline 05/07/1993 & 6.35 & 34.24 & 0.097 & 0.023 & 0.017 & 0.081 & 0.043 & 0.026 & 0.090 & 0.169 \\
\hline 19/07/1993 & 6.33 & 34.34 & 0.095 & 0.023 & 0.017 & 0.081 & 0.043 & 0.035 & 0.090 & 0.168 \\
\hline 02/08/1993 & 6.60 & 34.04 & 0.097 & 0.023 & 0.017 & 0.087 & 0.044 & 0.032 & 0.089 & 0.169 \\
\hline $17 / 08 / 1993$ & 6.55 & 35.70 & 0.098 & 0.023 & 0.017 & 0.084 & 0.046 & 0.052 & 0.091 & 0.166 \\
\hline $30 / 08 / 1993$ & 6.50 & 35.30 & 0.098 & 0.023 & 0.017 & 0.083 & 0.046 & 0.048 & 0.091 & 0.167 \\
\hline $27 / 09 / 1993$ & 6.59 & 36.25 & 0.103 & 0.023 & 0.018 & 0.084 & 0.047 & 0.052 & 0.089 & 0.166 \\
\hline $11 / 10 / 1993$ & 6.54 & 36.05 & 0.097 & 0.021 & 0.018 & 0.094 & 0.044 & 0.054 & 0.090 & 0.165 \\
\hline $25 / 10 / 1993$ & 6.45 & 40.15 & 0.103 & 0.024 & 0.021 & 0.098 & 0.050 & 0.066 & 0.102 & 0.158 \\
\hline 08/11/1993 & 6.48 & 37.05 & 0.103 & 0.023 & 0.019 & 0.096 & 0.050 & 0.060 & 0.101 & 0.160 \\
\hline 23/11/1993 & 6.51 & 37.05 & 0.101 & 0.023 & 0.019 & 0.087 & 0.046 & 0.054 & 0.095 & 0.163 \\
\hline 06/12/1993 & 6.29 & 34.04 & 0.103 & 0.023 & 0.018 & 0.089 & 0.046 & 0.051 & 0.094 & 0.163 \\
\hline $04 / 01 / 1994$ & 6.31 & 41.05 & 0.093 & 0.024 & 0.021 & 0.107 & 0.052 & 0.062 & 0.111 & 0.136 \\
\hline $31 / 01 / 1994$ & 6.23 & 42.05 & 0.095 & 0.024 & 0.021 & 0.100 & 0.049 & 0.056 & 0.108 & 0.141 \\
\hline 07/03/1994 & 6.33 & 40.05 & 0.096 & 0.023 & 0.019 & 0.098 & 0.045 & 0.049 & 0.099 & 0.150 \\
\hline 28/03/1994 & 6.25 & 37.05 & 0.095 & 0.022 & 0.019 & 0.095 & 0.049 & 0.053 & 0.106 & 0.150 \\
\hline $13 / 04 / 1994$ & 6.28 & 34.04 & 0.098 & 0.021 & 0.018 & 0.089 & 0.047 & 0.045 & 0.097 & 0.154 \\
\hline $25 / 04 / 1994$ & 6.25 & 38.05 & 0.097 & 0.024 & 0.019 & 0.097 & 0.046 & 0.054 & 0.104 & 0.147 \\
\hline 06/05/1994 & 6.42 & 38.05 & 0.097 & 0.024 & 0.018 & 0.090 & 0.045 & 0.049 & 0.097 & 0.154 \\
\hline $18 / 05 / 1994$ & 6.33 & 38.75 & 0.093 & 0.022 & 0.018 & 0.085 & 0.044 & 0.038 & 0.097 & 0.154 \\
\hline $24 / 05 / 1994$ & 6.29 & 38.05 & 0.098 & 0.023 & 0.018 & 0.091 & 0.044 & 0.049 & 0.094 & 0.154 \\
\hline $06 / 06 / 1994$ & 6.38 & 37.05 & 0.097 & 0.023 & 0.018 & 0.092 & 0.045 & 0.052 & 0.098 & 0.154 \\
\hline $20 / 06 / 1994$ & 6.34 & 36.00 & 0.098 & 0.023 & 0.018 & 0.089 & 0.049 & 0.050 & 0.100 & 0.156 \\
\hline 05/07/1994 & 6.58 & 36.96 & 0.095 & 0.022 & 0.017 & 0.086 & 0.043 & 0.043 & 0.095 & 0.158 \\
\hline $18 / 07 / 1994$ & 6.41 & 37.32 & 0.095 & 0.022 & 0.017 & 0.084 & 0.043 & 0.042 & 0.094 & 0.159 \\
\hline 01/08/1994 & 6.54 & 37.32 & 0.096 & 0.022 & 0.017 & 0.086 & 0.043 & 0.043 & 0.092 & 0.161 \\
\hline $16 / 08 / 1994$ & 6.22 & 37.14 & 0.097 & 0.023 & 0.017 & 0.083 & 0.047 & 0.043 & 0.091 & 0.158 \\
\hline 29/08/1994 & 6.31 & 37.14 & 0.097 & 0.023 & 0.017 & 0.084 & 0.045 & 0.043 & 0.092 & 0.162 \\
\hline $13 / 09 / 1994$ & 6.29 & 36.23 & 0.098 & 0.022 & 0.017 & 0.084 & 0.044 & 0.045 & 0.092 & 0.163 \\
\hline $27 / 09 / 1994$ & 6.40 & 36.23 & 0.095 & 0.023 & 0.017 & 0.082 & 0.043 & 0.044 & 0.089 & 0.164 \\
\hline $11 / 10 / 1994$ & 6.63 & 34.42 & 0.087 & 0.021 & 0.021 & 0.079 & 0.045 & 0.041 & 0.088 & 0.028 \\
\hline $25 / 10 / 1994$ & 6.26 & 36.23 & 0.097 & 0.023 & 0.017 & 0.084 & 0.044 & 0.044 & 0.091 & 0.164 \\
\hline 08/11/1994 & 6.34 & 35.33 & 0.098 & 0.022 & 0.016 & 0.085 & 0.043 & 0.044 & 0.092 & 0.166 \\
\hline $22 / 11 / 1994$ & 6.28 & 35.52 & 0.100 & 0.022 & 0.017 & 0.081 & 0.044 & 0.044 & 0.091 & 0.166 \\
\hline $06 / 12 / 1994$ & 6.60 & 37.41 & 0.099 & 0.022 & 0.018 & 0.086 & 0.046 & 0.053 & 0.091 & 0.167 \\
\hline 20/12/1994 & 6.13 & 38.41 & 0.099 & 0.023 & 0.019 & 0.092 & 0.046 & 0.057 & 0.099 & 0.160 \\
\hline 03/01/1995 & 6.47 & 38.32 & 0.098 & 0.022 & 0.019 & 0.090 & 0.046 & 0.053 & 0.097 & 0.160 \\
\hline 10/01/1995 & 6.18 & 37.68 & 0.099 & 0.023 & 0.018 & 0.088 & 0.047 & 0.054 & 0.097 & 0.161 \\
\hline
\end{tabular}




\begin{tabular}{|c|c|c|c|c|c|c|c|c|c|c|}
\hline 28/02/1995 & 6.00 & 41.67 & 0.095 & 0.023 & 0.020 & 0.093 & 0.048 & 0.061 & 0.102 & 0.151 \\
\hline 24/03/1995 & 5.99 & 42.76 & 0.096 & 0.025 & 0.021 & 0.100 & 0.051 & 0.075 & 0.103 & 0.146 \\
\hline $28 / 03 / 1995$ & 6.30 & 42.12 & 0.094 & 0.024 & 0.020 & 0.101 & 0.050 & 0.075 & 0.098 & 0.143 \\
\hline $25 / 04 / 1995$ & 6.55 & 38.23 & 0.094 & 0.022 & 0.018 & 0.087 & 0.047 & 0.054 & 0.090 & 0.154 \\
\hline 23/05/1995 & 6.21 & 38.41 & 0.095 & 0.022 & 0.018 & 0.087 & 0.045 & 0.053 & 0.092 & 0.158 \\
\hline $31 / 05 / 1995$ & 6.52 & 39.58 & 0.097 & 0.023 & 0.018 & 0.088 & 0.045 & 0.061 & 0.090 & 0.156 \\
\hline 06/06/1995 & 5.92 & 43.48 & 0.098 & 0.024 & 0.022 & 0.104 & 0.050 & 0.083 & 0.098 & 0.149 \\
\hline 20/06/1995 & 5.96 & 39.86 & 0.097 & 0.023 & 0.019 & 0.094 & 0.047 & 0.059 & 0.093 & 0.154 \\
\hline 04/07/1995 & 6.48 & 38.23 & 0.097 & 0.023 & 0.018 & 0.086 & 0.044 & 0.052 & 0.091 & 0.157 \\
\hline $18 / 07 / 1995$ & 6.48 & 37.95 & 0.096 & 0.022 & 0.018 & 0.084 & 0.045 & 0.048 & 0.091 & 0.159 \\
\hline $25 / 07 / 1995$ & 6.45 & 37.77 & 0.094 & 0.022 & 0.018 & 0.083 & 0.045 & 0.048 & 0.090 & 0.162 \\
\hline 01/08/1995 & 6.56 & 37.41 & 0.093 & 0.021 & 0.018 & 0.083 & 0.045 & 0.047 & 0.089 & 0.161 \\
\hline $15 / 08 / 1995$ & 6.53 & 37.77 & 0.098 & 0.023 & 0.017 & 0.081 & 0.044 & 0.046 & 0.090 & 0.162 \\
\hline 29/08/1995 & 6.53 & 37.95 & 0.097 & 0.024 & 0.017 & 0.083 & 0.044 & 0.047 & 0.090 & 0.164 \\
\hline 12/09/1995 & 6.46 & 37.50 & 0.096 & 0.022 & 0.017 & 0.081 & 0.045 & 0.046 & 0.089 & 0.167 \\
\hline 23/07/1995 & 6.40 & 37.50 & 0.095 & 0.022 & 0.018 & 0.084 & 0.044 & 0.033 & 0.090 & 0.157 \\
\hline 26/09/1995 & 6.47 & 38.68 & 0.097 & 0.023 & 0.018 & 0.086 & 0.048 & 0.059 & 0.090 & 0.166 \\
\hline 09/10/1995 & 6.15 & 38.41 & 0.094 & 0.024 & 0.020 & 0.078 & 0.049 & 0.038 & 0.089 & 0.165 \\
\hline $24 / 10 / 1995$ & 6.54 & 38.95 & 0.098 & 0.023 & 0.018 & 0.086 & 0.046 & 0.057 & 0.090 & 0.165 \\
\hline 07/11/1995 & 6.33 & 38.05 & 0.098 & 0.023 & 0.017 & 0.082 & 0.045 & 0.046 & 0.084 & 0.168 \\
\hline $21 / 11 / 1995$ & 6.30 & 39.68 & 0.098 & 0.023 & 0.017 & 0.087 & 0.046 & 0.062 & 0.084 & 0.167 \\
\hline 06/12/1995 & 6.39 & 39.22 & 0.103 & 0.025 & 0.019 & 0.090 & 0.050 & 0.068 & 0.089 & 0.166 \\
\hline $13 / 12 / 1995$ & 6.53 & 40.13 & 0.101 & 0.024 & 0.019 & 0.090 & 0.049 & 0.064 & 0.089 & 0.164 \\
\hline 19/12/1995 & 6.29 & 43.30 & 0.101 & 0.024 & 0.018 & 0.086 & 0.047 & 0.061 & 0.088 & 0.166 \\
\hline 02/01/1995 & 6.32 & 43.21 & 0.101 & 0.024 & 0.020 & 0.098 & 0.052 & 0.079 & 0.092 & 0.160 \\
\hline $16 / 01 / 1996$ & 6.29 & 39.58 & 0.097 & 0.023 & 0.019 & 0.088 & 0.050 & 0.067 & 0.089 & 0.161 \\
\hline $30 / 01 / 1996$ & 6.34 & 39.58 & 0.097 & 0.023 & 0.018 & 0.086 & 0.048 & 0.061 & 0.087 & 0.163 \\
\hline $23 / 04 / 1996$ & 6.32 & 37.77 & 0.101 & 0.023 & 0.018 & 0.089 & 0.048 & 0.064 & 0.091 & 0.166 \\
\hline 07/05/1996 & 6.33 & 36.87 & 0.100 & 0.023 & 0.018 & 0.086 & 0.047 & 0.060 & 0.089 & 0.168 \\
\hline $22 / 05 / 1996$ & 6.40 & 35.33 & 0.101 & 0.023 & 0.019 & 0.090 & 0.048 & 0.072 & 0.085 & 0.164 \\
\hline 04/06/1996 & 6.24 & 40.76 & 0.101 & 0.025 & 0.020 & 0.103 & 0.052 & 0.088 & 0.094 & 0.156 \\
\hline $18 / 06 / 1996$ & 6.30 & 38.05 & 0.100 & 0.024 & 0.019 & 0.095 & 0.051 & 0.075 & 0.089 & 0.160 \\
\hline 02/07/1996 & 6.30 & 38.05 & 0.096 & 0.025 & 0.021 & 0.085 & 0.051 & 0.053 & 0.086 & 0.156 \\
\hline $31 / 07 / 1996$ & 6.32 & 38.05 & 0.100 & 0.024 & 0.019 & 0.094 & 0.052 & 0.075 & 0.088 & 0.161 \\
\hline $13 / 08 / 1996$ & 6.20 & 38.05 & 0.101 & 0.024 & 0.019 & 0.091 & 0.050 & 0.068 & 0.089 & 0.164 \\
\hline 10/09/1996 & 6.43 & 36.87 & 0.099 & 0.023 & 0.018 & 0.084 & 0.050 & 0.059 & 0.087 & 0.166 \\
\hline $23 / 09 / 1996$ & 6.35 & 36.23 & 0.100 & 0.023 & 0.018 & 0.087 & 0.048 & 0.057 & 0.087 & 0.170 \\
\hline 08/10/1996 & 6.20 & 36.23 & 0.103 & 0.024 & 0.018 & 0.086 & 0.049 & 0.058 & 0.088 & 0.171 \\
\hline $21 / 10 / 1996$ & 6.15 & 40.05 & 0.098 & 0.025 & 0.021 & 0.083 & 0.051 & 0.054 & 0.082 & 0.168 \\
\hline $04 / 11 / 1996$ & 6.12 & 40.05 & 0.103 & 0.024 & 0.019 & 0.096 & 0.050 & 0.083 & 0.085 & 0.163 \\
\hline $12 / 02 / 1997$ & 6.31 & 36.05 & 0.096 & 0.024 & 0.021 & 0.086 & 0.053 & 0.058 & 0.086 & 0.157 \\
\hline $25 / 02 / 1997$ & 6.22 & 38.05 & 0.100 & 0.023 & 0.019 & 0.092 & 0.050 & 0.073 & 0.088 & 0.160 \\
\hline $11 / 03 / 1997$ & 6.28 & 40.05 & 0.099 & 0.024 & 0.020 & 0.097 & 0.053 & 0.084 & 0.092 & 0.153 \\
\hline $25 / 03 / 1997$ & 6.32 & 39.05 & 0.100 & 0.024 & 0.019 & 0.093 & 0.053 & 0.074 & 0.090 & 0.154 \\
\hline 08/04/1997 & 6.25 & 39.05 & 0.100 & 0.024 & 0.020 & 0.096 & 0.053 & 0.081 & 0.087 & 0.156 \\
\hline 21/04/1997 & 6.20 & 35.05 & 0.091 & 0.020 & 0.020 & 0.082 & 0.051 & 0.055 & 0.083 & 0.142 \\
\hline 06/05/1997 & 6.43 & 37.05 & 0.098 & 0.023 & 0.019 & 0.093 & 0.049 & 0.076 & 0.085 & 0.159 \\
\hline 20/05/1997 & 6.34 & 38.05 & 0.099 & 0.024 & 0.020 & 0.094 & 0.050 & 0.080 & 0.087 & 0.158 \\
\hline 03/06/1997 & 6.37 & 38.75 & 0.098 & 0.023 & 0.018 & 0.089 & 0.050 & 0.072 & 0.087 & 0.156 \\
\hline 17/06/1997 & 6.39 & 35.85 & 0.092 & 0.020 & 0.020 & 0.080 & 0.049 & 0.056 & 0.082 & 0.152 \\
\hline $01 / 07 / 1997$ & 6.33 & 39.35 & 0.099 & 0.024 & 0.019 & 0.094 & 0.051 & 0.081 & 0.082 & 0.154 \\
\hline $15 / 07 / 1997$ & 6.00 & 39.55 & 0.100 & 0.024 & 0.019 & 0.095 & 0.052 & 0.084 & 0.084 & 0.156 \\
\hline $29 / 07 / 1997$ & 6.41 & 37.05 & 0.099 & 0.023 & 0.019 & 0.094 & 0.050 & 0.079 & 0.085 & 0.154 \\
\hline $12 / 08 / 1997$ & 6.49 & 39.05 & 0.100 & 0.024 & 0.018 & 0.095 & 0.050 & 0.078 & 0.087 & 0.155 \\
\hline $26 / 08 / 1997$ & 6.57 & 36.25 & 0.095 & 0.022 & 0.020 & 0.080 & 0.051 & 0.057 & 0.081 & 0.156 \\
\hline 09/09/1997 & 6.55 & 38.35 & 0.099 & 0.023 & 0.018 & 0.087 & 0.048 & 0.068 & 0.084 & 0.165 \\
\hline 23/09/1997 & 6.47 & 38.05 & 0.100 & 0.023 & 0.018 & 0.087 & 0.050 & 0.068 & 0.086 & 0.162 \\
\hline 18/11/1997 & 6.20 & 36.05 & 0.098 & 0.025 & 0.021 & 0.086 & 0.053 & 0.061 & 0.082 & 0.159 \\
\hline $24 / 02 / 1998$ & 6.65 & 38.05 & 0.101 & 0.024 & 0.019 & 0.093 & 0.051 & 0.081 & 0.085 & 0.165 \\
\hline 21/04/1998 & 6.54 & 38.05 & 0.097 & 0.023 & 0.019 & 0.093 & 0.053 & 0.077 & 0.086 & 0.165 \\
\hline
\end{tabular}




\begin{tabular}{|c|c|c|c|c|c|c|c|c|c|c|}
\hline 19/05/1998 & 6.49 & 39.05 & 0.098 & 0.023 & 0.019 & 0.090 & 0.051 & 0.071 & 0.084 & 0.163 \\
\hline 29/06/1999 & 6.49 & 33.00 & 0.096 & 0.023 & 0.018 & 0.086 & 0.048 & 0.060 & 0.082 & 0.158 \\
\hline $13 / 07 / 1999$ & 6.37 & 32.60 & 0.088 & 0.023 & 0.019 & 0.078 & 0.050 & 0.042 & 0.077 & 0.146 \\
\hline 27/07/1999 & 6.61 & 35.70 & 0.095 & 0.023 & 0.018 & 0.089 & 0.049 & 0.069 & 0.083 & 0.159 \\
\hline 10/08/1999 & 6.35 & 34.10 & 0.095 & 0.023 & 0.017 & 0.084 & 0.047 & 0.059 & 0.082 & 0.160 \\
\hline 24/08/1999 & 5.93 & 34.50 & 0.090 & 0.021 & 0.020 & 0.077 & 0.054 & 0.053 & 0.076 & 0.149 \\
\hline 06/09/1999 & 6.51 & 34.30 & 0.089 & 0.021 & 0.019 & 0.077 & 0.053 & 0.047 & 0.076 & 0.154 \\
\hline 21/09/1999 & 6.62 & 36.10 & 0.095 & 0.024 & 0.020 & 0.079 & 0.053 & 0.049 & 0.078 & 0.167 \\
\hline $13 / 06 / 2000$ & 6.57 & 35.00 & 0.096 & 0.024 & 0.018 & 0.084 & 0.049 & 0.064 & 0.083 & 0.161 \\
\hline $16 / 10 / 2000$ & 6.56 & 35.00 & 0.096 & 0.023 & 0.018 & 0.086 & 0.047 & 0.063 & 0.079 & 0.162 \\
\hline $30 / 10 / 2000$ & 6.35 & 35.00 & 0.099 & 0.023 & 0.018 & 0.086 & 0.049 & 0.066 & 0.082 & 0.164 \\
\hline $19 / 02 / 2001$ & 6.46 & 39.00 & 0.096 & 0.023 & 0.018 & 0.089 & 0.048 & 0.067 & 0.080 & 0.153 \\
\hline $20 / 03 / 2001$ & 6.49 & 39.10 & 0.090 & 0.023 & 0.019 & 0.092 & 0.048 & 0.073 & 0.085 & 0.141 \\
\hline $16 / 07 / 2001$ & 6.60 & 36.60 & 0.094 & 0.022 & 0.017 & 0.084 & 0.047 & 0.060 & 0.079 & 0.159 \\
\hline $23 / 07 / 2001$ & 6.52 & 34.70 & 0.094 & 0.023 & 0.017 & 0.083 & 0.046 & 0.061 & 0.077 & 0.159 \\
\hline 20/08/2001 & 6.63 & 36.70 & 0.096 & 0.023 & 0.017 & 0.082 & 0.048 & 0.058 & 0.078 & 0.162 \\
\hline $17 / 09 / 2001$ & 6.57 & 36.60 & 0.094 & 0.023 & 0.017 & 0.084 & 0.045 & 0.061 & 0.074 & 0.159 \\
\hline 05/10/2004 & 6.37 & 32.60 & 0.097 & 0.020 & 0.016 & 0.078 & 0.047 & 0.061 & 0.070 & 0.164 \\
\hline $13 / 12 / 2004$ & 6.65 & 36.00 & 0.097 & 0.022 & 0.017 & 0.083 & 0.050 & 0.071 & 0.071 & 0.159 \\
\hline $29 / 03 / 2005$ & 6.11 & 35.70 & 0.090 & 0.022 & 0.017 & 0.084 & 0.050 & 0.073 & 0.072 & 0.142 \\
\hline $03 / 05 / 2005$ & 6.42 & 33.60 & 0.094 & 0.021 & 0.017 & 0.083 & 0.051 & 0.067 & 0.069 & 0.153 \\
\hline $31 / 05 / 2005$ & 6.24 & 34.10 & 0.094 & 0.021 & 0.016 & 0.080 & 0.049 & 0.064 & 0.068 & 0.154 \\
\hline $11 / 07 / 2005$ & 6.31 & 32.90 & 0.091 & 0.020 & 0.015 & 0.077 & 0.047 & 0.056 & 0.065 & 0.156 \\
\hline $22 / 08 / 2005$ & 6.52 & 31.70 & 0.097 & 0.021 & 0.016 & 0.080 & 0.049 & 0.062 & 0.070 & 0.164 \\
\hline $03 / 10 / 2005$ & 6.58 & 33.10 & 0.096 & 0.022 & 0.016 & 0.079 & 0.050 & 0.065 & 0.068 & 0.163 \\
\hline $07 / 02 / 2006$ & 6.53 & 33.90 & 0.098 & 0.022 & 0.015 & 0.078 & 0.051 & 0.064 & 0.068 & 0.164 \\
\hline $03 / 04 / 2006$ & 6.22 & 33.40 & 0.084 & 0.021 & 0.017 & 0.082 & 0.048 & 0.074 & 0.067 & 0.126 \\
\hline $22 / 05 / 2006$ & 6.42 & 32.20 & 0.093 & 0.021 & 0.016 & 0.078 & 0.048 & 0.068 & 0.068 & 0.152 \\
\hline $10 / 07 / 2006$ & 6.55 & 35.60 & 0.092 & 0.020 & 0.016 & 0.078 & 0.047 & 0.057 & 0.066 & 0.158 \\
\hline $21 / 08 / 2006$ & 6.44 & 33.00 & 0.095 & 0.021 & 0.016 & 0.078 & 0.048 & 0.070 & 0.066 & 0.159 \\
\hline $02 / 10 / 2006$ & 6.40 & 36.50 & 0.094 & 0.021 & 0.017 & 0.082 & 0.050 & 0.080 & 0.065 & 0.151 \\
\hline $19 / 03 / 2007$ & 5.89 & 34.50 & 0.092 & 0.021 & 0.016 & 0.077 & 0.049 & 0.071 & 0.065 & 0.154 \\
\hline $16 / 04 / 2007$ & 6.17 & 34.30 & 0.090 & 0.021 & 0.016 & 0.078 & 0.048 & 0.072 & 0.065 & 0.154 \\
\hline $29 / 05 / 2007$ & 6.59 & 33.80 & 0.094 & 0.021 & 0.016 & 0.078 & 0.048 & 0.071 & 0.061 & 0.154 \\
\hline $23 / 07 / 2007$ & 6.58 & 33.70 & 0.093 & 0.020 & 0.015 & 0.078 & 0.048 & 0.071 & 0.061 & 0.156 \\
\hline $02 / 09 / 2007$ & 6.69 & 33.60 & 0.096 & 0.021 & 0.016 & 0.078 & 0.050 & 0.072 & 0.063 & 0.158 \\
\hline $15 / 10 / 2007$ & 6.48 & 34.10 & 0.096 & 0.021 & 0.016 & 0.078 & 0.050 & 0.069 & 0.062 & 0.163 \\
\hline $26 / 11 / 2007$ & 6.64 & 38.30 & 0.085 & 0.029 & 0.039 & 0.084 & 0.052 & 0.059 & 0.063 & 0.146 \\
\hline $21 / 01 / 2008$ & 6.54 & 34.00 & 0.094 & 0.021 & 0.017 & 0.080 & 0.049 & 0.068 & 0.061 & 0.157 \\
\hline $03 / 03 / 2008$ & 6.42 & 34.50 & 0.094 & 0.021 & 0.016 & 0.077 & 0.048 & 0.068 & 0.060 & 0.161 \\
\hline $14 / 04 / 2008$ & 6.48 & 33.40 & 0.089 & 0.021 & 0.017 & 0.083 & 0.046 & 0.087 & 0.062 & 0.136 \\
\hline $19 / 05 / 2008$ & 6.45 & 32.40 & 0.093 & 0.021 & 0.016 & 0.078 & 0.051 & 0.072 & 0.063 & 0.158 \\
\hline $22 / 05 / 2008$ & 6.33 & 31.50 & 0.094 & 0.020 & 0.016 & 0.076 & 0.051 & 0.069 & 0.060 & 0.157 \\
\hline $09 / 06 / 2008$ & 6.67 & 32.60 & 0.093 & 0.020 & 0.015 & 0.077 & 0.047 & 0.069 & 0.061 & 0.156 \\
\hline $04 / 08 / 2008$ & 6.76 & 31.20 & 0.093 & 0.019 & 0.017 & 0.071 & 0.046 & 0.056 & 0.059 & 0.163 \\
\hline $16 / 09 / 2008$ & 6.43 & 34.00 & 0.094 & 0.020 & 0.019 & 0.078 & 0.045 & 0.080 & 0.055 & 0.153 \\
\hline $27 / 10 / 2008$ & 6.60 & 35.20 & 0.095 & 0.022 & 0.017 & 0.078 & 0.047 & 0.079 & 0.056 & 0.158 \\
\hline $08 / 12 / 2008$ & 6.59 & 33.80 & 0.098 & 0.021 & 0.017 & 0.079 & 0.049 & 0.080 & 0.057 & 0.161 \\
\hline $19 / 01 / 2009$ & 6.54 & 34.60 & 0.096 & 0.021 & 0.016 & 0.071 & 0.048 & 0.069 & 0.055 & 0.159 \\
\hline $16 / 03 / 2009$ & 6.60 & 34.30 & 0.097 & 0.021 & 0.016 & 0.080 & 0.049 & 0.080 & 0.055 & 0.160 \\
\hline $11 / 05 / 2009$ & 6.63 & 35.60 & 0.098 & 0.022 & 0.017 & 0.084 & 0.053 & 0.094 & 0.054 & 0.153 \\
\hline $06 / 07 / 2009$ & 6.65 & 34.80 & 0.098 & 0.020 & 0.017 & 0.082 & 0.051 & 0.088 & 0.054 & 0.164 \\
\hline $01 / 09 / 2009$ & 6.61 & 36.20 & 0.101 & 0.019 & 0.016 & 0.076 & 0.053 & 0.079 & 0.055 & 0.166 \\
\hline $06 / 10 / 2009$ & 6.50 & 35.40 & 0.102 & 0.019 & 0.017 & 0.076 & 0.055 & 0.074 & 0.058 & 0.169 \\
\hline $08 / 12 / 2009$ & 6.69 & 35.70 & 0.103 & 0.020 & 0.017 & 0.082 & 0.051 & 0.099 & 0.051 & 0.168 \\
\hline $19 / 03 / 2010$ & 6.66 & 37.70 & 0.104 & 0.023 & 0.019 & 0.088 & 0.055 & 0.123 & 0.050 & 0.164 \\
\hline $30 / 03 / 2010$ & 6.39 & 34.10 & 0.102 & 0.024 & 0.022 & 0.104 & 0.059 & 0.174 & 0.045 & 0.147 \\
\hline $11 / 05 / 2010$ & 6.59 & 39.40 & 0.103 & 0.023 & 0.020 & 0.085 & 0.057 & 0.133 & 0.047 & 0.157 \\
\hline $24 / 05 / 2011$ & 6.66 & 36.70 & 0.112 & 0.021 & 0.02 & 0.071 & 0.062 & 0.138 & 0.045 & 0.163 \\
\hline 05/07/2011 & 6.44 & 39.00 & 0.109 & 0.018 & 0.021 & 0.088 & 0.058 & 0.147 & 0.043 & 0.176 \\
\hline
\end{tabular}




\begin{tabular}{|l|l|l|l|l|l|l|l|l|l|l|}
\hline $02 / 08 / 2011$ & 6.65 & 39.00 & 0.111 & 0.023 & 0.023 & 0.087 & 0.059 & 0.158 & 0.041 & 0.178 \\
\hline $30 / 08 / 2011$ & 6.68 & 38.80 & 0.108 & 0.020 & 0.021 & 0.094 & 0.060 & 0.149 & 0.042 & 0.175 \\
\hline $04 / 10 / 2011$ & 6.65 & 40.30 & 0.112 & 0.017 & 0.020 & 0.090 & 0.059 & 0.143 & 0.041 & 0.179 \\
\hline $25 / 10 / 2011$ & 6.77 & 40.30 & 0.112 & 0.023 & 0.026 & 0.083 & 0.059 & 0.150 & 0.043 & 0.179 \\
\hline $29 / 11 / 2011$ & 6.80 & 40.00 & 0.114 & 0.024 & 0.025 & 0.083 & 0.060 & 0.149 & 0.042 & 0.181 \\
\hline $05 / 05 / 2015$ & 6.53 & 33.90 & 0.085 & 0.019 & 0.017 & 0.091 & 0.039 & 0.104 & 0.046 & 0.141 \\
\hline $26 / 08 / 2015$ & 6.30 & 39.00 & 0.106 & 0.015 & 0.017 & 0.083 & 0.048 & 0.098 & 0.044 & 0.166 \\
\hline $10 / 11 / 2015$ & 6.70 & 37.50 & 0.105 & 0.019 & 0.019 & 0.092 & 0.052 & 0.121 & 0.047 & 0.165 \\
\hline
\end{tabular}

Table S3: chemical data for the CS3 spring from 1986 to 2015. 


\begin{tabular}{|c|c|c|c|c|c|c|c|c|c|c|}
\hline Date & $\mathrm{PH}$ & $\begin{array}{c}\text { Conductivity } \\
(\mu \mathrm{S} / \mathrm{cm})\end{array}$ & $\begin{array}{c}\mathrm{Na}^{+} \\
(\mathrm{mmol} / \mathrm{L})\end{array}$ & $\begin{array}{c}\mathrm{K}^{+} \\
(\mathrm{mmol} / \mathrm{L})\end{array}$ & $\begin{array}{c}\mathrm{Mg}^{2+} \\
(\mathrm{mmol} / \mathrm{L})\end{array}$ & $\begin{array}{c}\mathrm{Ca}^{2+} \\
(\mathrm{mmol} / \mathrm{L})\end{array}$ & $\begin{array}{c}\mathrm{Cl}^{-} \\
(\mathrm{mmol} / \mathrm{L})\end{array}$ & $\begin{array}{c}\mathrm{NO}_{3}^{-} \\
(\mathrm{mmol} / \mathrm{L})\end{array}$ & $\begin{array}{c}\mathrm{SO}_{4}{ }^{2-} \\
(\mathrm{mmol} / \mathrm{L})\end{array}$ & $\begin{array}{c}\mathrm{H}_{4} \mathrm{SiO}_{4} \\
(\mathrm{mmol} / \mathrm{L})\end{array}$ \\
\hline $14 / 01 / 1986$ & 6.23 & 53.00 & 0.088 & 0.034 & 0.032 & 0.124 & 0.064 & 0.094 & 0.120 & 0.122 \\
\hline $16 / 04 / 1986$ & 6.18 & 40.00 & 0.084 & 0.026 & 0.024 & 0.093 & 0.052 & 0.019 & 0.111 & 0.123 \\
\hline $14 / 05 / 1986$ & 5.63 & 41.00 & 0.083 & 0.025 & 0.024 & 0.090 & 0.049 & 0.000 & 0.110 & 0.124 \\
\hline $29 / 05 / 1986$ & 6.35 & 38.00 & 0.090 & 0.025 & 0.024 & 0.090 & 0.048 & 0.024 & 0.106 & 0.134 \\
\hline $19 / 06 / 1986$ & 6.21 & 38.00 & 0.088 & 0.025 & 0.024 & 0.088 & 0.047 & 0.022 & 0.105 & 0.130 \\
\hline $07 / 07 / 1986$ & 6.01 & 39.00 & 0.086 & 0.026 & 0.023 & 0.090 & 0.047 & 0.031 & 0.100 & 0.134 \\
\hline $28 / 07 / 1986$ & 5.88 & 40.00 & 0.094 & 0.025 & 0.023 & 0.090 & 0.049 & 0.031 & 0.102 & 0.152 \\
\hline $01 / 09 / 1986$ & 6.52 & 38.80 & 0.096 & 0.025 & 0.023 & 0.089 & 0.049 & 0.009 & 0.095 & 0.161 \\
\hline $15 / 09 / 1986$ & 6.34 & 39.30 & 0.095 & 0.025 & 0.022 & 0.090 & 0.048 & 0.014 & 0.093 & 0.156 \\
\hline $06 / 10 / 1986$ & 6.78 & 39.50 & 0.095 & 0.026 & 0.023 & 0.088 & 0.047 & 0.025 & 0.099 & 0.161 \\
\hline $27 / 10 / 1986$ & 6.38 & 39.50 & 0.086 & 0.028 & 0.023 & 0.093 & 0.050 & 0.044 & 0.101 & 0.139 \\
\hline $19 / 01 / 1987$ & 6.23 & 37.50 & 0.090 & 0.025 & 0.024 & 0.090 & 0.050 & 0.030 & 0.100 & 0.147 \\
\hline 27/11/1989 & 6.71 & 38.00 & 0.095 & 0.026 & 0.022 & 0.088 & 0.048 & 0.044 & 0.092 & 0.164 \\
\hline 27/12/1989 & 5.52 & 36.20 & 0.085 & 0.028 & 0.022 & 0.089 & 0.046 & 0.045 & 0.096 & 0.136 \\
\hline $08 / 01 / 1990$ & 5.98 & 38.50 & 0.090 & 0.026 & 0.021 & 0.092 & 0.050 & 0.045 & 0.097 & 0.153 \\
\hline $19 / 02 / 1990$ & 5.98 & 36.10 & 0.074 & 0.024 & 0.021 & 0.082 & 0.049 & 0.043 & 0.096 & 0.117 \\
\hline $13 / 08 / 1990$ & 6.81 & 38.00 & 0.096 & 0.024 & 0.020 & 0.087 & 0.052 & 0.038 & 0.091 & 0.169 \\
\hline $02 / 09 / 1991$ & 6.25 & 37.00 & 0.096 & 0.025 & 0.021 & 0.083 & 0.047 & 0.039 & 0.088 & 0.171 \\
\hline $23 / 09 / 1991$ & 6.51 & 36.30 & 0.095 & 0.025 & 0.021 & 0.085 & 0.049 & 0.038 & 0.088 & 0.165 \\
\hline $25 / 04 / 1994$ & 6.08 & 34.00 & 0.083 & 0.024 & 0.020 & 0.081 & 0.046 & 0.032 & 0.096 & 0.123 \\
\hline $18 / 05 / 1994$ & 6.20 & 37.80 & 0.088 & 0.023 & 0.020 & 0.080 & 0.046 & 0.029 & 0.097 & 0.143 \\
\hline $06 / 12 / 1994$ & 6.58 & 38.23 & 0.098 & 0.024 & 0.021 & 0.087 & 0.047 & 0.045 & 0.091 & 0.166 \\
\hline $24 / 03 / 1995$ & 5.54 & 35.96 & 0.082 & 0.024 & 0.021 & 0.081 & 0.049 & 0.042 & 0.092 & 0.123 \\
\hline $31 / 05 / 1995$ & 6.53 & 37.23 & 0.087 & 0.025 & 0.021 & 0.081 & 0.048 & 0.043 & 0.088 & 0.137 \\
\hline $18 / 07 / 1995$ & 6.47 & 37.50 & 0.093 & 0.024 & 0.020 & 0.080 & 0.046 & 0.040 & 0.089 & 0.154 \\
\hline $23 / 07 / 1995$ & 6.58 & 37.50 & 0.090 & 0.023 & 0.021 & 0.080 & 0.048 & 0.041 & 0.088 & 0.157 \\
\hline $25 / 07 / 1995$ & 6.38 & 37.32 & 0.091 & 0.023 & 0.021 & 0.080 & 0.046 & 0.022 & 0.085 & 0.151 \\
\hline $13 / 12 / 1995$ & 6.57 & 38.77 & 0.096 & 0.025 & 0.021 & 0.083 & 0.052 & 0.045 & 0.088 & 0.162 \\
\hline $13 / 12 / 2004$ & 6.58 & 35.70 & 0.096 & 0.024 & 0.020 & 0.078 & 0.056 & 0.071 & 0.071 & 0.152 \\
\hline $03 / 05 / 2005$ & 6.30 & 30.40 & 0.085 & 0.021 & 0.017 & 0.070 & 0.051 & 0.062 & 0.062 & 0.128 \\
\hline $31 / 05 / 2005$ & 6.29 & 32.70 & 0.089 & 0.021 & 0.018 & 0.072 & 0.050 & 0.062 & 0.064 & 0.141 \\
\hline $11 / 07 / 2005$ & 6.39 & 33.60 & 0.094 & 0.021 & 0.018 & 0.075 & 0.051 & 0.059 & 0.064 & 0.156 \\
\hline $22 / 08 / 2005$ & 6.68 & 31.90 & 0.097 & 0.022 & 0.019 & 0.077 & 0.052 & 0.064 & 0.065 & 0.163 \\
\hline $07 / 02 / 2006$ & 6.45 & 34.30 & 0.100 & 0.027 & 0.017 & 0.073 & 0.054 & 0.065 & 0.066 & 0.161 \\
\hline $03 / 04 / 2006$ & 5.69 & 32.50 & 0.080 & 0.024 & 0.019 & 0.070 & 0.054 & 0.088 & 0.059 & 0.105 \\
\hline $22 / 05 / 2006$ & 6.37 & 31.80 & 0.092 & 0.022 & 0.018 & 0.073 & 0.050 & 0.070 & 0.065 & 0.142 \\
\hline $10 / 07 / 2006$ & 6.60 & 35.50 & 0.092 & 0.021 & 0.018 & 0.073 & 0.050 & 0.055 & 0.064 & 0.155 \\
\hline $21 / 08 / 2006$ & 6.47 & 33.20 & 0.091 & 0.024 & 0.018 & 0.070 & 0.050 & 0.071 & 0.060 & 0.145 \\
\hline $02 / 10 / 2006$ & 6.50 & 33.50 & 0.087 & 0.024 & 0.018 & 0.070 & 0.054 & 0.065 & 0.061 & 0.140 \\
\hline $19 / 03 / 2007$ & 5.68 & 33.70 & 0.090 & 0.022 & 0.018 & 0.069 & 0.052 & 0.063 & 0.061 & 0.143 \\
\hline $16 / 04 / 2007$ & 5.56 & 34.60 & 0.086 & 0.022 & 0.017 & 0.068 & 0.049 & 0.058 & 0.062 & 0.140 \\
\hline $19 / 05 / 2008$ & 6.06 & 32.40 & 0.093 & 0.022 & 0.018 & 0.072 & 0.054 & 0.075 & 0.058 & 0.146 \\
\hline $11 / 05 / 2009$ & 6.30 & 38.40 & 0.098 & 0.021 & 0.021 & 0.083 & 0.056 & 0.111 & 0.050 & 0.140 \\
\hline $06 / 07 / 2009$ & 6.64 & 35.10 & 0.098 & 0.022 & 0.020 & 0.080 & 0.053 & 0.103 & 0.048 & 0.154 \\
\hline $06 / 10 / 2009$ & 6.69 & 37.30 & 0.104 & 0.024 & 0.022 & 0.076 & 0.056 & 0.095 & 0.055 & 0.172 \\
\hline $08 / 12 / 2009$ & 6.64 & 37.90 & 0.102 & 0.023 & 0.023 & 0.087 & 0.055 & 0.137 & 0.047 & 0.159 \\
\hline $19 / 03 / 2010$ & 6.28 & 37.40 & 0.102 & 0.024 & 0.022 & 0.085 & 0.057 & 0.133 & 0.048 & 0.151 \\
\hline $30 / 03 / 2010$ & 6.07 & 38.40 & 0.094 & 0.024 & 0.023 & 0.083 & 0.057 & 0.169 & 0.042 & 0.124 \\
\hline $29 / 03 / 2011$ & 6.38 & 35.60 & 0.100 & 0.023 & 0.022 & 0.072 & 0.052 & 0.109 & 0.049 & 0.155 \\
\hline $24 / 05 / 2011$ & 6.48 & 33.00 & 0.101 & 0.021 & 0.02 & 0.069 & 0.051 & 0.099 & 0.048 & 0.161 \\
\hline $05 / 07 / 2011$ & 6.39 & 36.00 & 0.101 & 0.022 & 0.021 & 0.077 & 0.052 & 0.116 & 0.050 & 0.175 \\
\hline $02 / 08 / 2011$ & 6.87 & 34.00 & 0.097 & 0.024 & 0.022 & 0.077 & 0.049 & 0.120 & 0.042 & 0.174 \\
\hline $30 / 08 / 2011$ & 6.64 & 34.00 & 0.099 & 0.023 & 0.020 & 0.073 & 0.050 & 0.109 & 0.046 & 0.170 \\
\hline $04 / 10 / 2011$ & 6.84 & 36.30 & 0.102 & 0.030 & 0.022 & 0.064 & 0.051 & 0.101 & 0.047 & 0.177 \\
\hline $25 / 10 / 2011$ & 6.39 & 35.90 & 0.108 & 0.023 & 0.019 & 0.068 & 0.053 & 0.109 & 0.047 & 0.182 \\
\hline $29 / 11 / 2011$ & 6.51 & 35.70 & 0.106 & 0.025 & 0.024 & 0.066 & 0.052 & 0.103 & 0.050 & 0.188 \\
\hline $05 / 05 / 2015$ & 6.01 & 27.38 & 0.071 & 0.020 & 0.015 & 0.061 & 0.034 & 0.072 & 0.041 & 0.120 \\
\hline $26 / 08 / 2015$ & 6.39 & 37.30 & 0.096 & 0.021 & 0.017 & 0.067 & 0.044 & 0.071 & 0.045 & 0.172 \\
\hline
\end{tabular}




\begin{tabular}{|l|l|l|l|l|l}
\hline $10 / 11 / 2015$ & 6.70 & 31.98 & 0.096 & 0.018 & 0.017 \\
\hline
\end{tabular}

Table S4: chemical data for the CS4 spring from 1986 to 2015. 


\begin{tabular}{|c|c|c|c|c|c|c|c|c|c|c|}
\hline Date & $\mathrm{PH}$ & $\begin{array}{c}\text { Conductivity } \\
(\mu \mathrm{S} / \mathrm{cm})\end{array}$ & $\begin{array}{c}\mathrm{Na}^{+} \\
(\mathrm{mmol} / \mathrm{L})\end{array}$ & $\begin{array}{c}\mathrm{K}^{+} \\
(\mathrm{mmol} / \mathrm{L})\end{array}$ & $\begin{array}{c}\mathrm{Mg}^{2+} \\
(\mathrm{mmol} / \mathrm{L})\end{array}$ & $\begin{array}{c}\mathrm{Ca}^{2+} \\
(\mathrm{mmol} / \mathrm{L})\end{array}$ & $\begin{array}{c}\mathrm{Cl}^{-} \\
(\mathrm{mmol} / \mathrm{L})\end{array}$ & $\begin{array}{c}\mathrm{NO}_{3}^{-} \\
(\mathrm{mmol} / \mathrm{L})\end{array}$ & $\begin{array}{c}\mathrm{SO}_{4}{ }^{2-} \\
(\mathrm{mmol} / \mathrm{L})\end{array}$ & $\begin{array}{c}\mathrm{H}_{4} \mathrm{SiO}_{4} \\
(\mathrm{mmol} / \mathrm{L})\end{array}$ \\
\hline $06 / 10 / 1986$ & 6.56 & 59.20 & 0.000 & 0.101 & 0.041 & 0.047 & 0.078 & 0.076 & 0.138 & 0.137 \\
\hline $24 / 11 / 1989$ & 6.40 & 51.00 & 0.000 & 0.097 & 0.035 & 0.043 & 0.065 & 0.091 & 0.123 & 0.146 \\
\hline $20 / 02 / 1990$ & 6.64 & 47.00 & 0.001 & 0.088 & 0.026 & 0.050 & - & 0.043 & 0.105 & 0.132 \\
\hline $06 / 12 / 1994$ & 6.63 & 48.28 & 0.001 & 0.094 & 0.031 & 0.038 & 0.059 & 0.081 & 0.115 & 0.147 \\
\hline $31 / 05 / 1995$ & 6.42 & 48.92 & 0.001 & 0.089 & 0.031 & 0.037 & 0.063 & 0.085 & 0.112 & 0.129 \\
\hline $18 / 07 / 1995$ & 6.52 & 49.55 & 0.001 & 0.092 & 0.031 & 0.039 & 0.062 & 0.083 & 0.111 & 0.140 \\
\hline $12 / 07 / 2004$ & 5.89 & 46.50 & 0.000 & 0.092 & 0.030 & 0.036 & 0.060 & 0.119 & 0.080 & 0.144 \\
\hline $28 / 09 / 2004$ & 6.03 & 50.50 & 0.000 & 0.098 & 0.031 & 0.040 & 0.060 & 0.158 & 0.076 & 0.146 \\
\hline $02 / 11 / 2004$ & 6.34 & 41.20 & 0.000 & 0.089 & 0.031 & 0.035 & 0.073 & 0.112 & 0.080 & 0.133 \\
\hline $13 / 12 / 2004$ & 6.54 & 44.70 & 0.000 & 0.091 & 0.030 & 0.035 & 0.069 & 0.111 & 0.111 & 0.141 \\
\hline $24 / 01 / 2005$ & 6.21 & 39.70 & 0.000 & 0.084 & 0.028 & 0.031 & 0.066 & 0.085 & 0.080 & 0.124 \\
\hline $07 / 03 / 2005$ & 6.34 & 39.80 & 0.000 & 0.087 & 0.028 & 0.031 & 0.063 & 0.093 & 0.081 & 0.136 \\
\hline $29 / 03 / 2005$ & 6.23 & 40.30 & 0.000 & 0.083 & 0.028 & 0.031 & 0.058 & 0.087 & 0.081 & 0.117 \\
\hline $03 / 05 / 2005$ & 6.26 & 38.20 & 0.000 & 0.082 & 0.027 & 0.030 & 0.058 & 0.079 & 0.081 & 0.127 \\
\hline $31 / 05 / 2005$ & 6.40 & 38.80 & 0.000 & 0.082 & 0.024 & 0.030 & 0.054 & 0.086 & 0.076 & 0.129 \\
\hline $11 / 07 / 2005$ & 6.53 & 41.10 & 0.000 & 0.086 & 0.027 & 0.031 & 0.055 & 0.095 & 0.075 & 0.138 \\
\hline $22 / 08 / 2005$ & 6.61 & 39.50 & 0.000 & 0.090 & 0.029 & 0.034 & 0.055 & 0.107 & 0.075 & 0.143 \\
\hline $03 / 10 / 2005$ & 6.56 & 40.80 & 0.000 & 0.088 & 0.030 & 0.033 & 0.057 & 0.098 & 0.076 & 0.145 \\
\hline $07 / 02 / 2006$ & 6.28 & 38.50 & 0.002 & 0.086 & 0.028 & 0.030 & 0.060 & 0.080 & 0.081 & 0.144 \\
\hline $03 / 04 / 2006$ & 6.30 & 41.90 & 0.000 & 0.085 & 0.030 & 0.031 & 0.054 & 0.107 & 0.074 & 0.105 \\
\hline $22 / 05 / 2006$ & 6.39 & 37.20 & 0.000 & 0.085 & 0.028 & 0.030 & 0.050 & 0.094 & 0.075 & 0.129 \\
\hline $10 / 07 / 2006$ & 6.55 & 42.20 & 0.000 & 0.086 & 0.028 & 0.031 & 0.053 & 0.082 & 0.077 & 0.139 \\
\hline $21 / 08 / 2006$ & 6.40 & 60.30 & 0.000 & 0.090 & 0.029 & 0.032 & 0.054 & 0.119 & 0.067 & 0.135 \\
\hline $02 / 10 / 2006$ & 6.48 & 42.40 & 0.000 & 0.088 & 0.032 & 0.033 & 0.059 & 0.115 & 0.071 & 0.132 \\
\hline $19 / 03 / 2007$ & 5.85 & 41.20 & 0.000 & 0.085 & 0.028 & 0.031 & 0.057 & 0.109 & 0.073 & 0.132 \\
\hline $16 / 04 / 2007$ & 5.85 & 43.10 & 0.000 & 0.084 & 0.028 & 0.031 & 0.057 & 0.104 & 0.074 & 0.130 \\
\hline $29 / 05 / 2007$ & 6.44 & 41.80 & 0.000 & 0.086 & 0.029 & 0.032 & 0.061 & 0.114 & 0.072 & 0.129 \\
\hline $23 / 07 / 2007$ & 6.20 & 42.60 & 0.000 & 0.087 & 0.031 & 0.033 & 0.058 & 0.117 & 0.070 & 0.137 \\
\hline $02 / 09 / 2007$ & 6.38 & 43.60 & 0.001 & 0.093 & 0.031 & 0.036 & 0.059 & 0.127 & 0.072 & 0.142 \\
\hline $15 / 10 / 2007$ & 6.21 & 45.30 & 0.000 & 0.063 & 0.031 & 0.036 & 0.059 & 0.127 & 0.068 & 0.147 \\
\hline $26 / 11 / 2007$ & 6.46 & 47.50 & 0.000 & 0.084 & 0.020 & 0.065 & 0.038 & 0.115 & 0.079 & 0.138 \\
\hline $21 / 01 / 2008$ & 6.33 & 41.80 & 0.000 & 0.087 & 0.028 & 0.033 & 0.055 & 0.110 & 0.072 & 0.137 \\
\hline $03 / 03 / 2008$ & 6.06 & 42.90 & 0.000 & 0.086 & 0.028 & 0.032 & 0.054 & 0.111 & 0.072 & 0.141 \\
\hline $14 / 04 / 2008$ & 6.45 & 40.60 & 0.000 & 0.085 & 0.029 & 0.033 & 0.056 & 0.129 & 0.071 & 0.114 \\
\hline $09 / 06 / 2008$ & 6.61 & 43.40 & 0.000 & 0.088 & 0.029 & 0.034 & 0.053 & 0.159 & 0.063 & 0.128 \\
\hline $04 / 08 / 2008$ & 6.78 & 43.60 & 0.000 & 0.094 & 0.029 & 0.038 & 0.051 & 0.133 & 0.067 & 0.147 \\
\hline $16 / 09 / 2008$ & 6.40 & 47.20 & 0.000 & 0.093 & 0.031 & 0.038 & 0.053 & 0.175 & 0.056 & 0.135 \\
\hline $27 / 10 / 2008$ & 6.75 & 43.60 & 0.000 & 0.090 & 0.029 & 0.034 & 0.053 & 0.141 & 0.059 & 0.140 \\
\hline $08 / 12 / 2008$ & 6.57 & 41.50 & 0.000 & 0.088 & 0.029 & 0.033 & 0.053 & 0.120 & 0.066 & 0.144 \\
\hline $19 / 01 / 2009$ & 6.44 & 41.00 & 0.000 & 0.088 & 0.028 & 0.032 & 0.053 & 0.107 & 0.068 & 0.144 \\
\hline $16 / 03 / 2009$ & 6.38 & 38.80 & 0.000 & 0.081 & 0.027 & 0.030 & 0.052 & 0.109 & 0.066 & 0.128 \\
\hline $11 / 05 / 2009$ & 6.51 & 38.20 & 0.000 & 0.084 & 0.027 & 0.031 & 0.047 & 0.096 & 0.065 & 0.126 \\
\hline $06 / 07 / 2009$ & 6.57 & 44.50 & 0.000 & 0.091 & 0.029 & 0.037 & 0.050 & 0.191 & 0.054 & 0.138 \\
\hline $01 / 09 / 2009$ & 6.70 & 45.50 & 0.000 & 0.093 & 0.030 & 0.032 & 0.051 & 0.129 & 0.067 & 0.152 \\
\hline $08 / 12 / 2009$ & 6.53 & 43.30 & 0.001 & 0.093 & 0.027 & 0.032 & 0.049 & 0.169 & 0.059 & 0.149 \\
\hline $19 / 03 / 2010$ & 6.26 & 38.90 & 0.001 & 0.082 & 0.026 & 0.030 & 0.050 & 0.109 & 0.063 & 0.133 \\
\hline $30 / 03 / 2010$ & 6.28 & 38.10 & 0.009 & 0.083 & 0.028 & 0.032 & 0.049 & 0.115 & 0.065 & 0.127 \\
\hline $29 / 03 / 2011$ & 6.55 & 31.60 & 0.084 & 0.027 & 0.032 & 0.074 & 0.047 & 0.073 & 0.068 & 0.144 \\
\hline $24 / 05 / 2011$ & 6.67 & 38.00 & 0.087 & 0.026 & 0.031 & 0.08 & 0.046 & 0.095 & 0.066 & 0.149 \\
\hline $30 / 08 / 2011$ & 6.75 & 36.00 & 0.083 & 0.027 & 0.030 & 0.083 & 0.042 & 0.096 & 0.062 & 0.159 \\
\hline $04 / 10 / 2011$ & 6.69 & 40.90 & 0.092 & 0.024 & 0.028 & 0.077 & 0.043 & 0.095 & 0.060 & 0.183 \\
\hline $29 / 11 / 2011$ & 6.90 & 40.40 & 0.095 & 0.030 & 0.036 & 0.076 & 0.044 & 0.098 & 0.063 & 0.171 \\
\hline
\end{tabular}

Table S5: chemical data for the RH3 spring from 1994 to 2011. 


\begin{tabular}{|c|c|c|c|c|c|c|c|c|c|c|}
\hline Date & PH & $\begin{array}{c}\text { Conductivity } \\
(\mu \mathrm{S} / \mathrm{cm})\end{array}$ & $\begin{array}{c}\mathrm{Na}^{+} \\
(\mathrm{mmol} / \mathrm{L})\end{array}$ & $\begin{array}{c}\mathrm{K}^{+} \\
(\mathrm{mmol} / \mathrm{L})\end{array}$ & $\begin{array}{c}\mathrm{Mg}^{2+} \\
(\mathrm{mmol} / \mathrm{L})\end{array}$ & $\begin{array}{c}\mathrm{Ca}^{2+} \\
(\mathrm{mmol} / \mathrm{L})\end{array}$ & $\begin{array}{c}\mathrm{Cl}^{-} \\
(\mathrm{mmol} / \mathrm{L})\end{array}$ & $\begin{array}{c}\mathrm{NO}_{3}^{-} \\
(\mathrm{mmol} / \mathrm{L})\end{array}$ & $\begin{array}{c}\mathrm{SO}_{4}^{2-} \\
(\mathrm{mmol} / \mathrm{L})\end{array}$ & $\begin{array}{c}\mathrm{H}_{4} \mathrm{SiO}_{4} \\
(\mathrm{mmol} / \mathrm{L})\end{array}$ \\
\hline $09 / 11 / 1992$ & 4.78 & & 0.055 & 0.009 & 0.004 & 0.010 & & & & \\
\hline $07 / 12 / 1992$ & 4.69 & & 0.043 & 0.011 & 0.004 & 0.006 & & & & \\
\hline $01 / 03 / 1993$ & 4.55 & & 0.040 & 0.012 & 0.005 & 0.010 & & & & \\
\hline 29/03/1993 & 4.71 & & 0.035 & 0.011 & 0.005 & 0.010 & & & & \\
\hline $14 / 09 / 1993$ & 4.86 & & 0.044 & 0.009 & 0.005 & 0.006 & & & & \\
\hline $11 / 10 / 1993$ & 4.81 & & 0.044 & 0.010 & 0.005 & 0.008 & & & & \\
\hline $08 / 11 / 1993$ & 4.79 & & 0.038 & 0.012 & 0.002 & 0.006 & & & & \\
\hline $06 / 12 / 1993$ & 4.96 & & 0.043 & 0.024 & 0.006 & 0.014 & & & & \\
\hline $04 / 01 / 1994$ & 4.81 & & 0.031 & 0.008 & 0.002 & 0.003 & & & & \\
\hline $31 / 01 / 1994$ & 4.89 & & 0.032 & 0.008 & 0.003 & 0.003 & & & & \\
\hline $28 / 03 / 1994$ & 4.86 & & 0.023 & 0.013 & 0.000 & 0.004 & & & & \\
\hline $25 / 04 / 1994$ & 4.83 & & 0.028 & 0.014 & 0.002 & 0.003 & & & & \\
\hline 24/05/1994 & 4.97 & & 0.032 & 0.041 & 0.002 & 0.005 & & & & \\
\hline $18 / 07 / 1994$ & 6.67 & & 0.036 & 0.301 & 0.016 & 0.028 & & & & \\
\hline $03 / 01 / 1995$ & 4.87 & & 0.039 & 0.010 & 0.002 & 0.005 & & & & \\
\hline $30 / 01 / 1995$ & 4.90 & & 0.036 & 0.000 & 0.001 & 0.002 & & & & \\
\hline $28 / 02 / 1995$ & 4.85 & & 0.036 & 0.004 & 0.001 & 0.005 & & & & \\
\hline $27 / 03 / 1995$ & 4.93 & & 0.034 & 0.000 & 0.001 & 0.004 & & & & \\
\hline $24 / 04 / 1995$ & 4.93 & & 0.037 & 0.007 & 0.002 & 0.006 & & & & \\
\hline $20 / 06 / 1995$ & 4.98 & & 0.042 & 0.003 & 0.002 & 0.005 & & & & \\
\hline $21 / 10 / 1997$ & 4.70 & & 0.050 & 0.009 & 0.002 & 0.007 & 0.073 & 0.008 & 0.042 & 0.113 \\
\hline $16 / 12 / 1997$ & 4.81 & & 0.043 & 0.013 & 0.002 & 0.005 & 0.035 & 0.007 & 0.049 & 0.100 \\
\hline $30 / 12 / 1997$ & 4.71 & & 0.042 & 0.005 & 0.002 & 0.004 & 0.039 & 0.000 & 0.046 & 0.098 \\
\hline $13 / 01 / 1998$ & 4.77 & & 0.046 & 0.008 & 0.002 & 0.004 & 0.042 & 0.007 & 0.043 & 0.090 \\
\hline $24 / 02 / 1998$ & 4.99 & & 0.049 & 0.052 & 0.008 & 0.013 & 0.057 & 0.019 & 0.039 & 0.038 \\
\hline $10 / 03 / 1998$ & 4.78 & & 0.037 & 0.031 & 0.003 & 0.006 & 0.043 & 0.007 & 0.036 & 0.056 \\
\hline $07 / 04 / 1998$ & 5.47 & & 0.031 & 0.067 & 0.014 & 0.025 & 0.043 & 0.026 & 0.044 & 0.005 \\
\hline $06 / 05 / 1998$ & 4.79 & & 0.027 & 0.025 & 0.004 & 0.008 & 0.029 & 0.034 & 0.023 & 0.040 \\
\hline $25 / 08 / 1998$ & 4.67 & & 0.018 & 0.063 & 0.010 & 0.019 & 0.020 & 0.063 & 0.014 & 0.011 \\
\hline $21 / 09 / 1998$ & 4.60 & & 0.059 & 0.014 & 0.003 & 0.006 & 0.069 & 0.017 & 0.038 & 0.112 \\
\hline $02 / 11 / 1998$ & 4.81 & & 0.054 & 0.005 & 0.002 & 0.003 & 0.038 & 0.004 & 0.041 & 0.104 \\
\hline $20 / 10 / 2003$ & 5.39 & 17.70 & 0.048 & 0.058 & 0.004 & 0.011 & 0.052 & 0.006 & 0.021 & 0.083 \\
\hline $03 / 11 / 2003$ & 5.89 & 63.50 & 0.048 & & 0.017 & 0.052 & 0.042 & 0.002 & 0.013 & 0.078 \\
\hline $02 / 11 / 2004$ & 4.82 & 15.80 & 0.062 & 0.002 & 0.001 & 0.002 & 0.049 & 0.001 & 0.030 & 0.106 \\
\hline $24 / 01 / 2005$ & 4.84 & 16.70 & 0.060 & 0.002 & 0.001 & 0.002 & 0.043 & 0.007 & 0.030 & 0.098 \\
\hline $07 / 03 / 2005$ & 4.80 & 21.30 & 0.073 & 0.002 & 0.001 & 0.002 & 0.071 & 0.026 & 0.027 & 0.096 \\
\hline $29 / 03 / 2005$ & 4.84 & 20.40 & 0.069 & 0.002 & 0.001 & 0.002 & 0.059 & 0.026 & 0.027 & 0.095 \\
\hline $03 / 05 / 2005$ & 4.76 & 16.80 & 0.064 & 0.001 & 0.001 & 0.001 & 0.046 & 0.016 & 0.027 & 0.099 \\
\hline $22 / 08 / 2005$ & 4.80 & 15.20 & 0.066 & 0.001 & 0.001 & 0.001 & 0.045 & 0.010 & 0.025 & 0.110 \\
\hline $03 / 10 / 2005$ & 4.78 & 17.30 & 0.067 & 0.002 & 0.001 & 0.002 & 0.051 & 0.007 & 0.031 & 0.110 \\
\hline $28 / 11 / 2005$ & 4.90 & 16.70 & 0.061 & 0.003 & 0.001 & 0.001 & 0.031 & 0.003 & 0.032 & 0.102 \\
\hline $07 / 02 / 2006$ & 4.83 & 18.70 & 0.060 & 0.003 & 0.001 & 0.002 & 0.034 & 0.021 & 0.029 & 0.088 \\
\hline $03 / 04 / 2006$ & 4.79 & 17.00 & 0.058 & 0.001 & 0.001 & 0.002 & 0.042 & 0.009 & 0.035 & 0.101 \\
\hline $22 / 05 / 2006$ & 4.78 & 15.70 & 0.052 & 0.002 & 0.001 & 0.007 & 0.029 & 0.009 & 0.038 & 0.099 \\
\hline $10 / 07 / 2006$ & 4.85 & 19.80 & 0.057 & 0.008 & 0.001 & 0.002 & 0.028 & 0.002 & 0.030 & 0.113 \\
\hline $21 / 08 / 2006$ & 4.85 & 18.90 & 0.058 & 0.003 & 0.001 & 0.001 & 0.039 & 0.006 & 0.027 & 0.110 \\
\hline $02 / 10 / 2006$ & 4.90 & 15.80 & 0.054 & 0.002 & 0.001 & 0.001 & 0.030 & 0.003 & 0.030 & 0.103 \\
\hline $22 / 01 / 2007$ & 4.90 & 14.70 & 0.050 & 0.002 & 0.001 & 0.001 & 0.035 & 0.002 & 0.026 & 0.099 \\
\hline $19 / 03 / 2007$ & 4.85 & 14.10 & 0.037 & 0.001 & 0.001 & 0.002 & 0.040 & 0.005 & 0.016 & 0.043 \\
\hline $16 / 04 / 2007$ & 4.82 & 15.10 & 0.040 & 0.002 & 0.001 & 0.002 & 0.034 & 0.005 & 0.018 & 0.078 \\
\hline $29 / 05 / 2007$ & 4.87 & 14.10 & 0.048 & 0.002 & 0.002 & 0.001 & 0.043 & 0.003 & 0.020 & 0.102 \\
\hline $23 / 07 / 2007$ & 4.85 & 14.40 & 0.052 & 0.002 & 0.001 & 0.004 & 0.048 & 0.005 & 0.019 & 0.104 \\
\hline $02 / 09 / 2007$ & 4.87 & 14.90 & 0.052 & 0.002 & 0.002 & 0.001 & 0.037 & 0.006 & 0.026 & 0.107 \\
\hline $26 / 11 / 2007$ & 4.75 & 19.30 & 0.059 & 0.001 & 0.001 & 0.003 & 0.065 & 0.014 & 0.021 & 0.072 \\
\hline $21 / 01 / 2008$ & 4.84 & 16.60 & 0.047 & 0.001 & 0.001 & 0.002 & 0.048 & 0.013 & 0.023 & 0.074 \\
\hline $03 / 03 / 2008$ & 4.80 & 15.40 & 0.045 & 0.001 & 0.001 & 0.002 & 0.044 & 0.003 & 0.022 & 0.076 \\
\hline $14 / 04 / 2008$ & 4.85 & 17.50 & 0.045 & 0.001 & 0.001 & 0.001 & 0.042 & 0.008 & 0.022 & 0.070 \\
\hline $22 / 05 / 2008$ & 4.92 & 13.00 & 0.043 & 0.001 & 0.001 & 0.001 & 0.036 & 0.004 & 0.022 & 0.093 \\
\hline
\end{tabular}




\begin{tabular}{|l|c|c|c|c|c|c|c|c|c|c|}
\hline $09 / 06 / 2008$ & 4.92 & 15.10 & 0.051 & 0.001 & 0.001 & 0.001 & 0.049 & 0.002 & 0.019 & 0.094 \\
\hline $04 / 08 / 2008$ & - & - & - & - & - & - & - & - & - & - \\
\hline $16 / 09 / 2008$ & 4.96 & 16.20 & 0.059 & 0.002 & 0.004 & 0.001 & 0.054 & 0.004 & 0.020 & 0.101 \\
\hline $08 / 12 / 2008$ & 4.92 & 13.90 & 0.054 & 0.003 & 0.003 & 0.002 & 0.039 & 0.006 & 0.022 & 0.088 \\
\hline $19 / 01 / 2009$ & 4.91 & 15.70 & 0.036 & 0.001 & 0.001 & 0.001 & 0.028 & 0.015 & 0.016 & 0.042 \\
\hline $16 / 03 / 2009$ & 4.82 & 14.40 & 0.043 & 0.002 & 0.001 & 0.002 & 0.024 & 0.007 & 0.024 & 0.075 \\
\hline $11 / 05 / 2009$ & 4.87 & 15.00 & 0.050 & 0.001 & 0.000 & 0.001 & 0.037 & 0.008 & 0.021 & 0.098 \\
\hline $06 / 07 / 2009$ & 6.26 & 19.50 & 0.019 & 0.099 & 0.006 & 0.009 & 0.023 & 0.015 & 0.019 & 0.008 \\
\hline $01 / 09 / 2009$ & 4.74 & 16.60 & 0.062 & 0.002 & 0.002 & 0.001 & 0.039 & 0.006 & 0.022 & 0.108 \\
\hline $13 / 10 / 2009$ & 6.42 & 24.0 & 0.022 & 0.118 & 0.004 & 0.016 & 0.046 & 0.026 & 0.008 & 0.005 \\
\hline $08 / 12 / 2009$ & 4.95 & 15.6 & 0.066 & 0.006 & 0.002 & 0.003 & 0.058 & 0.005 & 0.018 & 0.092 \\
\hline $05 / 01 / 2010$ & 4.86 & 15.80 & 0.062 & 0.001 & 0.002 & 0.001 & 0.053 & 0.003 & 0.022 & 0.094 \\
\hline $02 / 03 / 2010$ & 4.87 & 16.10 & 0.067 & 0.002 & 0.002 & 0.003 & 0.049 & 0.009 & 0.023 & 0.095 \\
\hline $30 / 03 / 2010$ & 5.02 & 14.00 & 0.061 & 0.002 & 0.002 & 0.001 & 0.030 & 0.006 & 0.025 & 0.098 \\
\hline $25 / 05 / 2010$ & 4.86 & 13.50 & 0.058 & 0.002 & 0.003 & 0.002 & 0.026 & 0.007 & 0.024 & 0.106 \\
\hline $22 / 06 / 2010$ & 4.90 & 14.60 & 0.062 & 0.002 & 0.002 & 0.002 & 0.034 & 0.009 & 0.022 & 0.106 \\
\hline $17 / 08 / 2010$ & 4.99 & 15.40 & 0.065 & 0.001 & 0.003 & 0.001 & 0.047 & 0.005 & 0.020 & 0.108 \\
\hline $14 / 09 / 2010$ & 5.96 & 13.10 & 0.028 & 0.055 & 0.004 & 0.006 & 0.028 & 0.013 & 0.011 & 0.036 \\
\hline $07 / 12 / 2010$ & 4.88 & 19.20 & 0.060 & 0.002 & 0.001 & 0.007 & 0.035 & 0.004 & 0.022 & 0.096 \\
\hline $04 / 01 / 2011$ & 5.03 & 13.00 & 0.059 & 0.001 & 0.002 & 0.001 & 0.038 & 0.007 & 0.018 & 0.095 \\
\hline $01 / 02 / 2011$ & 5.01 & 11.20 & 0.047 & 0.001 & 0.002 & 0.001 & 0.019 & 0.005 & 0.020 & 0.100 \\
\hline $05 / 07 / 2011$ & 5.51 & 11.00 & 0.050 & 0.005 & 0.002 & 0.001 & 0.028 & 0.01 & 0.018 & 0.121 \\
\hline $30 / 08 / 2011$ & 4.96 & 11.40 & 0.060 & 0.003 & 0.002 & 0.001 & 0.042 & 0.006 & 0.018 & 0.124 \\
\hline
\end{tabular}

Table S6: chemical data for the HP-70 soil solution from 1990 to 2011. 


\begin{tabular}{|c|c|c|c|c|c|c|c|c|c|c|}
\hline Date & PH & $\begin{array}{c}\text { Conductivity } \\
(\mu \mathrm{S} / \mathrm{cm})\end{array}$ & $\begin{array}{c}\mathrm{Na}^{+} \\
(\mathrm{mmol} / \mathrm{L})\end{array}$ & $\begin{array}{c}\mathrm{K}^{+} \\
(\mathrm{mmol} / \mathrm{L})\end{array}$ & $\begin{array}{c}\mathrm{Mg}^{2+} \\
(\mathrm{mmol} / \mathrm{L})\end{array}$ & $\begin{array}{c}\mathrm{Ca}^{2+} \\
(\mathrm{mmol} / \mathrm{L})\end{array}$ & $\begin{array}{c}\mathrm{Cl}^{-} \\
(\mathrm{mmol} / \mathrm{L})\end{array}$ & $\begin{array}{c}\mathrm{NO}_{3}^{-} \\
(\mathrm{mmol} / \mathrm{L})\end{array}$ & $\begin{array}{c}\mathrm{SO}_{4}{ }^{2-} \\
(\mathrm{mmol} / \mathrm{L})\end{array}$ & $\begin{array}{c}\mathrm{H}_{4} \mathrm{SiO}_{4} \\
(\mathrm{mmol} \\
/ \mathrm{L})\end{array}$ \\
\hline $12 / 10 / 1992$ & 4.43 & & 0.050 & 0.055 & 0.010 & 0.032 & & & & \\
\hline $09 / 11 / 1992$ & 4.39 & & 0.053 & 0.029 & 0.009 & 0.025 & & & & \\
\hline $07 / 12 / 1992$ & 4.50 & & 0.046 & 0.036 & 0.007 & 0.019 & & & & \\
\hline 19/01/1993 & 4.44 & & 0.040 & 0.032 & 0.007 & 0.031 & & & & \\
\hline $01 / 03 / 1993$ & 4.43 & & 0.045 & 0.029 & 0.010 & 0.032 & & & & \\
\hline 29/03/1993 & 4.25 & & 0.076 & 0.036 & 0.014 & 0.037 & & & & \\
\hline $26 / 04 / 1993$ & 4.63 & & 0.056 & 0.015 & 0.008 & 0.023 & & & & \\
\hline $24 / 05 / 1993$ & 4.56 & & 0.056 & 0.043 & 0.009 & 0.036 & & & & \\
\hline $21 / 06 / 1993$ & 4.41 & & 0.053 & 0.046 & 0.013 & 0.038 & & & & \\
\hline $19 / 07 / 1993$ & 4.44 & & 0.047 & 0.038 & 0.010 & 0.031 & & & & \\
\hline $17 / 08 / 1993$ & 4.45 & & 0.057 & 0.048 & 0.011 & 0.049 & & & & \\
\hline $14 / 09 / 1993$ & 4.37 & & 0.061 & 0.040 & 0.012 & 0.033 & & & & \\
\hline $11 / 10 / 1993$ & 4.51 & & 0.065 & 0.036 & 0.011 & 0.028 & & & & \\
\hline $08 / 11 / 1993$ & 4.41 & & 0.056 & 0.028 & 0.008 & 0.025 & & & & \\
\hline $06 / 12 / 1993$ & 4.32 & & 0.050 & 0.040 & 0.012 & 0.032 & & & & \\
\hline 04/01/1994 & 4.40 & & 0.049 & 0.020 & 0.009 & 0.025 & & & & \\
\hline $31 / 01 / 1994$ & 4.51 & & 0.032 & 0.019 & 0.009 & 0.020 & & & & \\
\hline $24 / 02 / 1994$ & 4.52 & & 0.046 & 0.026 & 0.009 & 0.023 & & & & \\
\hline $28 / 03 / 1994$ & 4.58 & & 0.027 & 0.018 & 0.006 & 0.027 & & & & \\
\hline $25 / 04 / 1994$ & 4.29 & & 0.045 & 0.031 & 0.012 & 0.027 & & & & \\
\hline $24 / 05 / 1994$ & 4.53 & & 0.043 & 0.033 & 0.010 & 0.031 & & & & \\
\hline $21 / 06 / 1994$ & 4.56 & & 0.046 & 0.029 & 0.009 & 0.034 & & & & \\
\hline $16 / 08 / 1994$ & 4.32 & & 0.052 & 0.030 & 0.013 & 0.054 & & & & \\
\hline $12 / 09 / 1994$ & 4.42 & & 0.054 & 0.049 & 0.015 & 0.058 & & & & \\
\hline $10 / 10 / 1994$ & 3.94 & & 0.090 & 0.082 & 0.024 & 0.090 & & & & \\
\hline $05 / 12 / 1994$ & 4.28 & & 0.098 & 0.059 & 0.030 & 0.104 & & & & \\
\hline $03 / 01 / 1995$ & 4.31 & & 0.090 & 0.041 & 0.028 & 0.083 & & & & \\
\hline $30 / 01 / 1995$ & 4.33 & & 0.065 & 0.025 & 0.017 & 0.042 & & & & \\
\hline $28 / 02 / 1995$ & 4.54 & & 0.049 & 0.022 & 0.012 & 0.036 & & & & \\
\hline $27 / 03 / 1995$ & 4.36 & & 0.039 & 0.015 & 0.012 & 0.032 & & & & \\
\hline $24 / 04 / 1995$ & 4.48 & & 0.040 & 0.020 & 0.012 & 0.033 & & & & \\
\hline $23 / 05 / 1995$ & 4.65 & & 0.036 & 0.021 & 0.011 & 0.039 & & & & \\
\hline 20/06/1995 & 4.57 & & 0.042 & 0.038 & 0.011 & 0.032 & & & & \\
\hline $18 / 07 / 1995$ & 4.57 & & 0.046 & 0.027 & 0.011 & 0.051 & & & & \\
\hline 16/08/1995 & 4.43 & & 0.034 & 0.063 & 0.013 & 0.037 & & & & \\
\hline $12 / 09 / 1995$ & 4.45 & & 0.058 & 0.077 & 0.021 & 0.059 & & & & \\
\hline $21 / 10 / 1997$ & 4.41 & & 0.058 & 0.031 & 0.011 & 0.030 & 0.083 & 0.150 & 0.058 & 0.092 \\
\hline $18 / 11 / 1997$ & 4.61 & & 0.057 & 0.033 & 0.010 & 0.031 & 0.082 & 0.090 & 0.064 & 0.111 \\
\hline $02 / 12 / 1997$ & 4.65 & & 0.049 & 0.019 & 0.009 & 0.028 & 0.073 & 0.066 & 0.064 & 0.137 \\
\hline $16 / 12 / 1997$ & 4.41 & & 0.062 & 0.026 & 0.011 & 0.026 & 0.078 & 0.075 & 0.066 & 0.128 \\
\hline $30 / 12 / 1997$ & 4.31 & & 0.078 & 0.039 & 0.014 & 0.029 & 0.095 & 0.076 & 0.070 & 0.084 \\
\hline $13 / 01 / 1998$ & 4.45 & & 0.062 & 0.024 & 0.010 & 0.024 & 0.076 & 0.077 & 0.064 & 0.133 \\
\hline $24 / 02 / 1998$ & 4.45 & & 0.056 & 0.028 & 0.010 & 0.022 & 0.067 & 0.071 & 0.059 & 0.100 \\
\hline $10 / 03 / 1998$ & 4.43 & & 0.061 & 0.019 & 0.010 & 0.025 & 0.076 & 0.077 & 0.069 & 0.142 \\
\hline $23 / 03 / 1998$ & 4.46 & & 0.058 & 0.025 & 0.011 & 0.029 & 0.075 & 0.089 & 0.075 & 0.124 \\
\hline $07 / 04 / 1998$ & 5.04 & & 0.066 & 0.101 & 0.029 & 0.075 & 0.089 & 0.119 & 0.090 & 0.004 \\
\hline $21 / 04 / 1998$ & 4.46 & & 0.057 & 0.023 & 0.011 & 0.027 & 0.075 & 0.065 & 0.065 & 0.109 \\
\hline $06 / 05 / 1998$ & 4.45 & & 0.060 & 0.022 & 0.011 & 0.028 & 0.084 & 0.101 & 0.060 & 0.130 \\
\hline $16 / 06 / 1998$ & 4.47 & & 0.066 & 0.021 & 0.011 & 0.032 & 0.077 & 0.180 & 0.053 & 0.143 \\
\hline $01 / 07 / 1998$ & 4.54 & & 0.067 & 0.019 & 0.010 & 0.028 & 0.085 & 0.124 & 0.057 & 0.160 \\
\hline $11 / 08 / 1998$ & 4.33 & & 0.050 & 0.076 & 0.018 & 0.087 & 0.064 & 0.072 & 0.043 & 0.064 \\
\hline $25 / 08 / 1998$ & 3.98 & & 0.048 & 0.098 & 0.025 & 0.090 & 0.077 & 0.147 & 0.038 & 0.049 \\
\hline $08 / 09 / 1998$ & 3.87 & & 0.039 & 0.140 & 0.047 & 0.083 & 0.056 & 0.038 & 0.048 & 0.011 \\
\hline 21/09/1998 & 4.48 & & 0.057 & 0.023 & 0.008 & 0.025 & 0.067 & 0.119 & 0.054 & 0.111 \\
\hline $13 / 01 / 2003$ & - & & 0.107 & 0.014 & 0.016 & 0.008 & 0.057 & 0.081 & 0.135 & 0.055 \\
\hline $20 / 10 / 2003$ & 4.47 & 46.50 & 0.076 & 0.024 & 0.016 & 0.014 & 0.050 & 0.165 & 0.051 & 0.060 \\
\hline $02 / 11 / 2004$ & 4.28 & 88.60 & 0.158 & 0.016 & 0.026 & 0.009 & 0.272 & 0.385 & 0.049 & 0.083 \\
\hline
\end{tabular}




\begin{tabular}{|c|c|c|c|c|c|c|c|c|c|c|}
\hline $13 / 12 / 2004$ & 4.26 & 75.50 & 0.120 & 0.038 & 0.021 & 0.031 & 0.178 & 0.209 & 0.209 & 0.024 \\
\hline $24 / 01 / 2005$ & 4.32 & 93.90 & 0.155 & 0.012 & 0.030 & 0.009 & 0.124 & 0.536 & 0.053 & 0.069 \\
\hline $07 / 03 / 2005$ & 4.84 & 16.40 & 0.019 & 0.002 & 0.004 & 0.002 & 0.010 & 0.084 & 0.008 & 0.012 \\
\hline $29 / 03 / 2005$ & 4.38 & 83.20 & 0.099 & 0.010 & 0.025 & 0.010 & 0.039 & 0.513 & 0.052 & 0.084 \\
\hline $03 / 10 / 2005$ & 4.39 & 46.80 & 0.088 & 0.013 & 0.011 & 0.006 & 0.088 & 0.071 & 0.088 & 0.098 \\
\hline $28 / 11 / 2005$ & 4.60 & 45.60 & 0.095 & 0.014 & 0.013 & 0.009 & 0.098 & 0.084 & 0.087 & 0.094 \\
\hline $07 / 02 / 2006$ & 4.57 & 45.40 & 0.075 & 0.013 & 0.013 & 0.008 & 0.082 & 0.100 & 0.069 & 0.060 \\
\hline $03 / 04 / 2006$ & 4.31 & 53.50 & 0.100 & 0.010 & 0.016 & 0.012 & 0.075 & 0.157 & 0.085 & 0.071 \\
\hline $22 / 05 / 2006$ & 4.44 & 57.80 & 0.117 & 0.012 & 0.018 & 0.007 & 0.061 & 0.274 & 0.073 & 0.068 \\
\hline $10 / 07 / 2006$ & 4.40 & 69.20 & 0.111 & 0.010 & 0.018 & 0.006 & 0.059 & 0.277 & 0.067 & 0.083 \\
\hline $21 / 08 / 2006$ & 4.50 & 22.40 & 0.087 & 0.010 & 0.013 & 0.004 & 0.076 & 0.075 & 0.083 & 0.090 \\
\hline $02 / 10 / 2006$ & 4.52 & 44.00 & 0.074 & 0.013 & 0.011 & 0.003 & 0.086 & 0.020 & 0.087 & 0.058 \\
\hline $22 / 01 / 2007$ & 4.55 & 44.00 & 0.080 & 0.020 & 0.013 & 0.009 & 0.076 & 0.080 & 0.077 & 0.066 \\
\hline $19 / 03 / 2007$ & 4.48 & 49.20 & 0.072 & 0.011 & 0.016 & 0.004 & 0.083 & 0.124 & 0.072 & 0.061 \\
\hline $16 / 04 / 2007$ & 4.47 & 48.70 & 0.063 & 0.010 & 0.014 & 0.005 & 0.082 & 0.115 & 0.070 & 0.053 \\
\hline $29 / 05 / 2007$ & 4.45 & 38.40 & 0.063 & 0.010 & 0.013 & 0.004 & 0.061 & 0.039 & 0.084 & 0.067 \\
\hline $23 / 07 / 2007$ & 4.46 & 37.40 & 0.066 & 0.010 & 0.009 & 0.004 & 0.070 & 0.008 & 0.086 & 0.068 \\
\hline 02/09/2007 & 4.51 & 38.50 & 0.077 & 0.011 & 0.011 & 0.006 & 0.056 & 0.063 & 0.089 & 0.069 \\
\hline $15 / 10 / 2007$ & 4.49 & 46.90 & 0.087 & 0.042 & 0.020 & 0.024 & 0.059 & 0.058 & 0.105 & 0.063 \\
\hline $26 / 11 / 2007$ & 4.48 & 44.90 & 0.077 & 0.006 & 0.012 & 0.004 & 0.038 & 0.128 & 0.069 & 0.059 \\
\hline $21 / 01 / 2008$ & 4.53 & 45.70 & 0.084 & 0.008 & 0.013 & 0.005 & 0.042 & 0.148 & 0.069 & 0.053 \\
\hline 03/03/2008 & 4.42 & 51.50 & 0.084 & 0.007 & 0.014 & 0.004 & 0.030 & 0.207 & 0.064 & 0.058 \\
\hline $14 / 04 / 2008$ & 4.44 & 53.30 & 0.082 & 0.006 & 0.019 & 0.002 & 0.025 & 0.233 & 0.068 & 0.059 \\
\hline $22 / 05 / 2008$ & 4.53 & 39.40 & 0.068 & 0.005 & 0.012 & 0.003 & 0.029 & 0.133 & 0.070 & 0.065 \\
\hline 09/06/2008 & 4.65 & 37.60 & 0.065 & 0.011 & 0.009 & 0.003 & 0.035 & 0.066 & 0.075 & 0.066 \\
\hline $04 / 08 / 2008$ & - & - & - & - & - & - & - & - & - & - \\
\hline $16 / 09 / 2008$ & 4.53 & 38.80 & 0.076 & 0.006 & 0.012 & 0.002 & 0.050 & 0.076 & 0.062 & 0.071 \\
\hline $14 / 10 / 2008$ & 4.50 & 37.60 & 0.077 & 0.005 & 0.009 & 0.003 & 0.062 & 0.053 & 0.071 & 0.065 \\
\hline $08 / 12 / 2008$ & 4.56 & 35.10 & 0.078 & 0.004 & 0.011 & 0.003 & 0.058 & 0.036 & 0.071 & 0.063 \\
\hline $19 / 01 / 2009$ & 4.44 & 38.40 & 0.077 & 0.005 & 0.009 & 0.003 & 0.031 & 0.059 & 0.072 & 0.031 \\
\hline $16 / 03 / 2009$ & 4.55 & 39.50 & 0.092 & 0.009 & 0.010 & 0.004 & 0.025 & 0.071 & 0.088 & 0.051 \\
\hline $11 / 05 / 2009$ & 4.54 & 43.20 & 0.089 & 0.006 & 0.011 & 0.004 & 0.019 & 0.142 & 0.074 & 0.058 \\
\hline $06 / 07 / 2009$ & 4.52 & 47.10 & 0.099 & 0.027 & 0.014 & 0.011 & 0.048 & 0.161 & 0.061 & 0.055 \\
\hline 01/09/2009 & 4.60 & 39.10 & 0.097 & 0.009 & 0.009 & 0.003 & 0.059 & 0.072 & 0.067 & 0.073 \\
\hline $10 / 11 / 2009$ & 4.91 & 30.8 & 0.091 & 0.005 & 0.009 & 0.007 & 0.072 & 0.023 & 0.063 & 0.062 \\
\hline $08 / 12 / 2009$ & 4.67 & 39.1 & 0.115 & 0.008 & 0.01 & 0.005 & 0.135 & 0.01 & 0.073 & 0.063 \\
\hline $05 / 01 / 2010$ & 4.51 & 43.90 & 0.118 & 0.005 & 0.014 & 0.002 & 0.110 & 0.017 & 0.099 & 0.078 \\
\hline $02 / 03 / 2010$ & 4.67 & 29.60 & 0.080 & 0.003 & 0.009 & 0.002 & 0.032 & 0.014 & 0.080 & 0.056 \\
\hline $30 / 03 / 2010$ & 4.66 & 27.20 & 0.074 & 0.005 & 0.008 & 0.003 & 0.027 & 0.020 & 0.077 & 0.049 \\
\hline $25 / 05 / 2010$ & 4.55 & 30.20 & 0.083 & 0.003 & 0.008 & 0.002 & 0.016 & 0.009 & 0.088 & 0.058 \\
\hline $22 / 06 / 2010$ & 4.58 & 30.30 & 0.074 & 0.003 & 0.008 & 0.002 & 0.020 & 0.011 & 0.072 & 0.058 \\
\hline $17 / 08 / 2010$ & 4.67 & 32.90 & 0.086 & 0.003 & 0.009 & 0.002 & 0.060 & 0.012 & 0.070 & 0.066 \\
\hline $14 / 09 / 2010$ & 4.50 & 33.30 & 0.091 & 0.004 & 0.008 & 0.002 & 0.057 & 0.003 & 0.076 & 0.065 \\
\hline $09 / 11 / 2010$ & 4.67 & 32.00 & 0.099 & 0.007 & 0.009 & 0.002 & 0.059 & 0.011 & 0.076 & 0.057 \\
\hline $07 / 12 / 2010$ & 5.43 & 5.00 & 0.004 & 0.002 & 0.001 & 0.005 & 0.006 & 0.001 & 0.003 & 0.056 \\
\hline $04 / 01 / 2011$ & 4.62 & 31.40 & 0.090 & 0.002 & 0.008 & 0.002 & 0.024 & 0.006 & 0.095 & 0.056 \\
\hline $01 / 02 / 2011$ & 4.62 & 31.60 & 0.087 & 0.002 & 0.008 & 0.002 & 0.012 & 0.005 & 0.098 & 0.056 \\
\hline $29 / 03 / 2011$ & 4.67 & 27.00 & 0.078 & 0.004 & 0.008 & 0.004 & 0.014 & 0.003 & 0.085 & 0.051 \\
\hline $05 / 07 / 2011$ & 4.58 & 36.00 & 0.093 & 0.003 & 0.011 & 0.002 & 0.064 & 0.014 & 0.078 & 0.067 \\
\hline $30 / 08 / 2011$ & 4.56 & 30.40 & 0.077 & 0.003 & 0.008 & 0.001 & 0.046 & 0.003 & 0.073 & 0.067 \\
\hline $25 / 10 / 2011$ & 4.59 & 30.50 & 0.089 & 0.010 & 0.006 & 0.001 & 0.058 & 0.006 & 0.073 & 0.070 \\
\hline
\end{tabular}

Table S7: chemical data for the VP-60 soil solution from 1990 to 2011. 


\begin{tabular}{|c|c|c|c|c|c|c|c|c|c|c|}
\hline Date & $\mathrm{PH}$ & $\begin{array}{c}\text { Conductivity } \\
(\mu \mathrm{S} / \mathrm{cm})\end{array}$ & $\begin{array}{c}\mathrm{Na}^{+} \\
(\mathrm{mmol} / \mathrm{L})\end{array}$ & $\begin{array}{c}\mathrm{K}^{+} \\
(\mathrm{mmol} / \mathrm{L})\end{array}$ & $\begin{array}{c}\mathrm{Mg}^{2+} \\
(\mathrm{mmol} / \mathrm{L})\end{array}$ & $\begin{array}{c}\mathrm{Ca}^{2+} \\
(\mathrm{mmol} / \mathrm{L})\end{array}$ & $\begin{array}{c}\mathrm{Cl}^{-} \\
(\mathrm{mmol} / \mathrm{L})\end{array}$ & $\begin{array}{c}\mathrm{NO}_{3}^{-} \\
(\mathrm{mmol} / \mathrm{L})\end{array}$ & $\begin{array}{c}\mathrm{SO}_{4}^{2-} \\
(\mathrm{mmol} / \mathrm{L})\end{array}$ & $\begin{array}{c}\mathrm{H}_{4} \mathrm{SiO}_{4} \\
(\mathrm{mmol} / \mathrm{L})\end{array}$ \\
\hline $10 / 04 / 2015$ & 6.77 & 26.40 & 0.120 & 0.025 & 0.014 & 0.056 & 0.049 & 0.026 & 0.039 & 0.213 \\
\hline $05 / 05 / 2015$ & 6.29 & 21.85 & 0.074 & 0.013 & 0.011 & 0.053 & 0.028 & 0.021 & 0.034 & 0.153 \\
\hline $11 / 06 / 2015$ & 6.71 & 28.34 & 0.111 & 0.015 & 0.014 & 0.057 & 0.038 & 0.027 & 0.049 & 0.216 \\
\hline $26 / 08 / 2015$ & 5.88 & 22.85 & 0.121 & 0.017 & 0.014 & 0.056 & 0.041 & 0.041 & 0.050 & 0.220 \\
\hline $12 / 11 / 2015$ & 6.82 & 29.87 & 0.122 & 0.018 & 0.015 & 0.057 & 0.041 & 0.046 & 0.050 & 0.220 \\
\hline $02 / 12 / 2015$ & 6.14 & 19.29 & 0.074 & 0.013 & 0.009 & 0.033 & 0.031 & 0.020 & 0.036 & 0.143 \\
\hline
\end{tabular}

Table S8: chemical data for the PZ3 piezometer in 2015. 


\begin{tabular}{|c|c|c|c|c|c|c|c|c|c|c|}
\hline Date & $\mathrm{PH}$ & $\begin{array}{c}\text { Conductivity } \\
(\mu \mathrm{S} / \mathrm{cm})\end{array}$ & $\begin{array}{c}\mathrm{Na}^{+} \\
(\mathrm{mmol} / \mathrm{L})\end{array}$ & $\begin{array}{c}\mathrm{K}^{+} \\
(\mathrm{mmol} / \mathrm{L})\end{array}$ & $\begin{array}{c}\mathrm{Mg}^{2+} \\
(\mathrm{mmol} / \mathrm{L})\end{array}$ & $\begin{array}{c}\mathrm{Ca}^{2+} \\
(\mathrm{mmol} / \mathrm{L})\end{array}$ & $\begin{array}{c}\mathrm{Cl}^{-} \\
(\mathrm{mmol} / \mathrm{L})\end{array}$ & $\begin{array}{c}\mathrm{NO}_{3}^{-} \\
(\mathrm{mmol} / \mathrm{L})\end{array}$ & $\begin{array}{c}\mathrm{SO}_{4}{ }^{2-} \\
(\mathrm{mmol} / \mathrm{L})\end{array}$ & $\begin{array}{c}\mathrm{H}_{4} \mathrm{SiO}_{4} \\
(\mathrm{mmol} / \\
\mathrm{L})\end{array}$ \\
\hline $05 / 05 / 2015$ & 6.16 & 25.99 & 0.072 & 0.013 & 0.017 & 0.058 & 0.035 & 0.044 & 0.050 & 0.132 \\
\hline $04 / 06 / 2015$ & 6.55 & 26.85 & 0.081 & 0.013 & 0.019 & 0.064 & 0.038 & 0.034 & 0.050 & 0.154 \\
\hline $10 / 11 / 2015$ & 6.50 & 28.69 & 0.091 & 0.016 & 0.020 & 0.062 & 0.043 & 0.039 & 0.053 & 0.165 \\
\hline $12 / 11 / 2015$ & 6.72 & 28.73 & 0.089 & 0.015 & 0.020 & 0.060 & 0.043 & 0.038 & 0.052 & 0.165 \\
\hline $02 / 12 / 2015$ & 6.57 & 28.24 & 0.089 & 0.015 & 0.020 & 0.060 & 0.041 & 0.037 & 0.052 & 0.163 \\
\hline
\end{tabular}

Table S9: chemical data for the PZ5 piezometer in 2015. 


\begin{tabular}{|c|c|c|c|c|}
\hline Mineral & $\begin{array}{c}\text { Mass fraction } \\
\text { (HPT facies) } \\
(\%)\end{array}$ & $\begin{array}{c}\text { Reactive surface area } \\
(\mathrm{HPT} \text { facies }) \\
\left(\mathrm{m}^{2} / \mathrm{kg} \mathrm{H} \mathrm{O}\right)\end{array}$ & $\begin{array}{c}\text { Mass fraction } \\
\text { (CA facies) } \\
(\%)\end{array}$ & $\begin{array}{c}\text { Reactive surface area } \\
\text { (CA facies) } \\
\left(\mathrm{m}^{2} / \mathrm{kg} \mathrm{H}_{2} \mathrm{O}\right)\end{array}$ \\
\hline Quartz & 35 & 19.45 & 46 & 25.56 \\
\hline Albite & 31 & 12.92 & 5 & 0 \\
\hline K-feldspar & 22 & 5.16 & 0 & 0 \\
\hline Biotite & 6 & 10.23 & 48 & 81.84 \\
\hline Muscovite & 3 & 5.11 & 0 & 0 \\
\hline Anorthite & 2 & 0.83 & 0.3 & 0.004 \\
\hline Apatite & 0.5 & 0.02 & 0.7 & 26.26 \\
\hline Illite & 0.5 & 18.75 & 5 \\
\hline
\end{tabular}

Table S10: mineralogical compositions and reactive surfaces for the different bedrock facies used in this study (i.e., the HPT, CA). 


\begin{tabular}{|c|c|c|}
\hline Mineral & Chemical composition & $\log (\mathrm{K})\left(25^{\circ} \mathrm{C}\right)$ \\
\hline Quartz & $\mathrm{SiO}_{2}$ & -3.999 \\
\hline Albite & $\mathrm{NaAlSi}_{3} \mathrm{O}_{8}$ & -20.178 \\
\hline K-feldspath & $\mathrm{KAlSi}_{3} \mathrm{O}_{8}$ & -23.192 \\
\hline Biotite & $\mathrm{Si}_{3} \mathrm{AlMgFe}_{2} \mathrm{O}_{10}(\mathrm{OH})_{2} \mathrm{~K}$ & 9.482 \\
\hline Muscovite & $\mathrm{Si}_{3} \mathrm{Al}_{3} \mathrm{O}_{10}(\mathrm{OH})_{2} \mathrm{~K}$ & -53.169 \\
\hline Anorthite & $\mathrm{CaAl}_{2} \mathrm{Si}_{2} \mathrm{O}_{8}$ & -19.488 \\
\hline Apatite & $\mathrm{Ca}_{5}\left(\mathrm{PO}_{4}\right)_{3} \mathrm{~F}$ & -59.590 \\
\hline Illite & $\mathrm{Si}_{3.5} \mathrm{Al}_{2.1} \mathrm{Fe}_{0.34} \mathrm{Mg}_{0.04} \mathrm{O}_{10}(\mathrm{OH})_{2} \mathrm{~K}_{0.6}$ & -36.389 \\
\hline
\end{tabular}

Table S11: Chemical compositions and laboratory measured thermodynamic constants at $25^{\circ} \mathrm{C}$ for the different primary minerals used in this study. Data are from the KIRMAT database which is consistent with the Thermoddem database. 


\begin{tabular}{|c|c|c|c|c|c|c|c|c|c|}
\hline \multirow[t]{2}{*}{ Mineral } & \multirow[t]{2}{*}{ Chemical composition } & \multicolumn{3}{|c|}{ 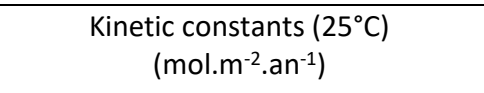 } & \multicolumn{2}{|c|}{$\alpha^{n_{H+}}$} & \multicolumn{2}{|c|}{$\begin{array}{c}\text { Boundary } \\
\mathrm{pH}\end{array}$} & \multirow{2}{*}{$\begin{array}{c}\text { Activation } \\
\text { energy } \\
\left(\mathrm{kJ} . \mathrm{mol}^{-1}\right)\end{array}$} \\
\hline & & Acid & Neutral & Basic & Acid & Basic & Acid & Basic & \\
\hline Quartz $^{(1)}$ & $\mathrm{SiO}_{2}$ & - & $3.15 \times 10^{-6}$ & $2.50 \times 10^{-9}$ & - & -0.5 & 3 & 6 & $85.0^{(1)}$ \\
\hline Albite ${ }^{(2)}$ & $\mathrm{NaAlSi}_{3} \mathrm{O}_{8}$ & $5.61 \times 10^{-3}$ & $5 \times 10^{-5}$ & $2.94 \times 10^{-7}$ & 0.45 & -0.3 & 4.5 & 8 & $58.6^{(12)}$ \\
\hline $\begin{array}{c}\text { K- } \\
\text { feldspath(3.4) }\end{array}$ & $\mathrm{KAISi}_{3} \mathrm{O}_{8}$ & $2.68 \times 10^{-3}$ & $1.02 \times 10^{-5}$ & $9.31 \times 10^{-13}$ & 0.44 & -0.69 & 4.5 & 10 & $38.0^{(13)}$ \\
\hline Biotite ${ }^{(5.6)}$ & $\mathrm{Si}_{3} \mathrm{AlMgFe}_{2} \mathrm{O}_{10}(\mathrm{OH})_{2} \mathrm{~K}$ & $4.56 \times 10^{-3}$ & $2.39 \times 10^{-5}$ & $1.09 \times 10^{-13}$ & 0.41 & -1.05 & 5 & 8 & $35.0^{(7)}$ \\
\hline Muscovite ${ }^{(7)}$ & $\mathrm{Si}_{3} \mathrm{Al}_{3} \mathrm{O}_{10}(\mathrm{OH})_{2} \mathrm{~K}$ & $1.99 \times 10^{-5}$ & - & $6.15 \times 10^{-5}$ & 0.17 & -0.16 & 4 & 7 & $22.0^{(7)}$ \\
\hline Anorthite ${ }^{(8.9)}$ & $\mathrm{CaAl}_{2} \mathrm{Si}_{2} \mathrm{O}_{8}$ & - & $3.23 \times 10^{-4}$ & - & - & - & 4 & 8.5 & $40.0^{(14)}$ \\
\hline Apatite $(10)$ & $\mathrm{Ca}_{5}\left(\mathrm{PO}_{4}\right)_{3} \mathrm{~F}$ & $5.50 \times 10^{2}$ & $2.96 \times 10^{-3}$ & - & 0.91 & - & 6 & - & $34.7^{(10)}$ \\
\hline Illite ${ }^{(11)}$ & $\mathrm{Si}_{3.5} \mathrm{Al}_{2.1} \mathrm{Fe}_{0.34} \mathrm{Mg}_{0.04} \mathrm{O}_{10}(\mathrm{OH})_{2} \mathrm{~K}_{0.6}$ & $7.92 \times 10^{-5}$ & $3.71 \times 10^{-8}$ & $3.88 \times 10^{-14}$ & 0.64 & -0.62 & 5 & 9.5 & $14.0^{(11)}$ \\
\hline
\end{tabular}

Table S12: Kinetic constants at $25^{\circ} \mathrm{C}$ and activation energies of the dissolution reactions for the primary minerals used in this study. Data are from laboratory measurements and from ${ }^{(1)}$ Dove (1994). (2) Chou and Wollast (1984). (3) Schweda (1989). (4) Lundström and Øhman (1990). (5) Acker and Bricker (1992). (6) Malmström and Banwart (1997). (7) Nagy (1995). (8) Amrhein and Suarez (1992). (9) Berg and Banwart (2000). (10) Guidry and Mackenzie (2003). ${ }^{(11)}$ Köhler et al. (2003). (12) Blum and Stillings (1995). ${ }^{(13)}$ Helgeson et al. (1984). ${ }^{(14)}$ Madé (1991). The acid, neutral, and basic domains correspond to the domains where hydrolysis reactions are promoted by $\mathrm{H}^{+}, \mathrm{H}_{2} \mathrm{O}$ and $\mathrm{OH}^{-}$respectively. 


\begin{tabular}{|c|c|c|c|}
\hline Mineral & Chemical composition & Log $(\mathrm{K})\left(25^{\circ} \mathrm{C}\right)$ & Log (Qi $/ \mathrm{Ki})$ \\
\hline K-Illite & $\mathrm{Si}_{3.5} \mathrm{Al}_{2.3} \mathrm{Mg}_{0.25} \mathrm{O}_{10}(\mathrm{OH})_{2} \mathrm{~K}_{0.6}$ & -43.192 & 22.40 \\
\hline Mg-Illite & $\mathrm{Si}_{3.43} \mathrm{Al}_{2.24} \mathrm{Mg}_{0.38} \mathrm{O}_{10}(\mathrm{OH})_{2} \mathrm{~K}_{0.8}$ & -38.760 & 7.95 \\
\hline Ca-Illite & $\mathrm{Si}_{3.2 .} \mathrm{Al}_{2.2} \mathrm{Mg}_{0.1} \mathrm{Fe}_{0.5} \mathrm{O}_{10}(\mathrm{OH})_{2} \mathrm{~K}_{0.6} \mathrm{Ca}_{0.15}$ & -40.943 & 9.22 \\
\hline Montmorillonite & $\mathrm{Si}_{3.83} \mathrm{Al}_{1.84} \mathrm{Mg}_{0.38} \mathrm{O}_{10}(\mathrm{OH})_{2} \mathrm{~K}_{0.4}$ & -35.723 & 12.18 \\
\hline Na-Montmorillonite & $\mathrm{Si}_{3.667} \mathrm{Al}_{2.333} \mathrm{O}_{10}(\mathrm{OH})_{2} \mathrm{Na}_{0.333}$ & -47.951 & 1.18 \\
\hline K-Montmorillonite & $\mathrm{Si}_{3.667} \mathrm{Al}_{2.333} \mathrm{O}_{10}(\mathrm{OH})_{2} \mathrm{~K}_{0.333}$ & -48.191 & 1.04 \\
\hline Ca-Montmorillonite & $\mathrm{Si}_{3.667} \mathrm{Al}_{2.333} \mathrm{O}_{10}(\mathrm{OH})_{2} \mathrm{Ca}_{0.167}$ & -48.101 & 0.13 \\
\hline Mg-Montmorillonite & $\mathrm{Si}_{3.667} \mathrm{Al}_{2.333} \mathrm{O}_{10}(\mathrm{OH})_{2} \mathrm{Mg}_{0.167}$ & -48.201 & 0.18 \\
\hline
\end{tabular}

Table S13: Chemical compositions and laboratory measured thermodynamic constants at $25^{\circ} \mathrm{C}$ for the different clay solid solution end members used in this study. Data are from the KIRMAT database which is consistent with the Thermoddem database. Log(Qi/Ki) values give the saturation states of the clay end members along a typical flow path in the watershed. 


\begin{tabular}{|c|c|c|c|}
\hline Mineral & $\log (\mathrm{Q} / \mathrm{K})$ & $\begin{array}{c}\text { Dissolution rate } \\
\text { (moles/yr/ kg H20) }\end{array}$ & $\begin{array}{c}\text { Precipitation rate } \\
\text { (moles/yr/ kg H20) }\end{array}$ \\
\hline Quartz* & 0.61 & 0 & 0 \\
\hline Albite* & -4.22 & $1.34 \mathrm{E}-4$ & 0 \\
\hline K-feldspar* & -1.62 & $1.29 \mathrm{E}-5$ & 0 \\
\hline Biotite* & -3.45 & $5.79 \mathrm{E}-5$ & 0 \\
\hline Muscovite* & 0.31 & 0 & 0 \\
\hline Anorthite* & -12.64 & $1.11 \mathrm{E}-5$ & 0 \\
\hline Apatite* & -40.40 & $1.85 \mathrm{E}-5$ & 0 \\
\hline Calcite & -3.88 & 0 & 0 \\
\hline Talc & -17.53 & 0 & 0 \\
\hline Magnesite & -4.83 & 0 & 0 \\
\hline Siderite & -2.03 & 0 & 0 \\
\hline Anhydrite & -4.55 & 0 & 0 \\
\hline Gypsum & -4.08 & 0 & 0 \\
\hline Amorphous silica & -0.81 & 0 & 0 \\
\hline Dolomite & -7.08 & 0 & 0 \\
\hline Hematite & 0.57 & 0 & 0 \\
\hline
\end{tabular}

Table S14: Mean values of $\log (\mathrm{Q} / \mathrm{K})$, dissolution and precipitation rates for the minerals used in the study. Values are given for a typical flow line in the Strengbach watershed. Minerals with a star are primary minerals present in the bedrock and identified by XRD and/or thin section observation. Minerals with no star are secondary minerals tested in precipitation. $Q$ is the ion activity product of the mineral and $K$ the thermodynamic equilibrium constant of the dissolution reaction.

\section{References}

Acker. J. G.. \& Bricker. O. P. (1992). The influence of $\mathrm{pH}$ on biotite dissolution and alteration kinetics at low temperature. Geochimica et Cosmochimica Acta. 56(8). 3073-3092.

Amrhein. C.. \& Suarez. D. L. (1992). Some factors affecting the dissolution kinetics of anorthite at 25 C. Geochimica et Cosmochimica Acta. 56(5). 1815-1826.

Berg. A.. \& Banwart. S. A. (2000). Carbon dioxide mediated dissolution of Ca-feldspar: implications for silicate weathering. Chemical Geology. 163(1). 25-42.

Blum. A. E.. \& Stillings. L. L. (1995). Feldspar dissolution kinetics. Reviews in Mineralogy and Geochemistry. 31(1). 291-351.

Chou. L.. \& Wollast. R. (1984). Study of the weathering of albite at room temperature and pressure with a fluidized bed reactor. Geochimica et Cosmochimica Acta. 48(11). 2205-2217.

Dove. P. M. (1994). The dissolution kinetics of quartz in sodium chloride solutions at 25 degrees to 300 degrees C. American Journal of Science. 294(6). 665-712. 
Guidry. M. W.. \& Mackenzie. F. T. (2003). Experimental study of igneous and sedimentary apatite dissolution: control of $\mathrm{pH}$. distance from equilibrium. and temperature on dissolution rates. Geochimica et Cosmochimica Acta. 67(16). 2949-2963.

Helgeson. H. C.. Murphy. W. M.. \& Aagaard. P. (1984). Thermodynamic and kinetic constraints on reaction rates among minerals and aqueous solutions. II. Rate constants. effective surface area. and the hydrolysis of feldspar. Geochimica et Cosmochimica Acta. 48(12). 2405-2432.

Köhler. S. J.. Dufaud. F.. \& Oelkers. E. H. (2003). An experimental study of illite dissolution kinetics as a function of $\mathrm{pH}$ from 1.4 to 12.4 and temperature from 5 to 50 C. Geochimica et Cosmochimica Acta. 67(19). 35833594.

Lundström. U.. \& Øhman. L. O. (1990). Dissolution of feldspars in the presence of natural. organic solutes. Journal of Soil Science. 41(3). 359-369.

Madé. B. (1991). Modélisation thermodynamique et cinétique des réactions géochimiques dans les interactions eau-roche (Doctoral dissertation).

Malmström. M.. \& Banwart. S. (1997). Biotite dissolution at 25 C: The pH dependence of dissolution rate and stoichiometry. Geochimica et Cosmochimica Acta. 61(14). 2779-2799.

Murphy. W. M.. \& Helgeson. H. C. (1987). Thermodynamic and kinetic constraints on reaction rates among minerals and aqueous solutions. III. Activated complexes and the $\mathrm{pH}$-dependence of the rates of feldspar. pyroxene. wollastonite. and olivine hydrolysis. Geochimica et Cosmochimica Acta. 51(12). 3137-3153.

Nagy. K. L.. White. A. F.. \& Brantley. S. L. (1995). Chemical weathering rates of silicate minerals. Mineralogical Society of America. Washington. DC.

Schweda. P. (1989). Kinetics of alkali feldspar dissolution at low temperature. Ph.D. dissertation Stockholm University. 